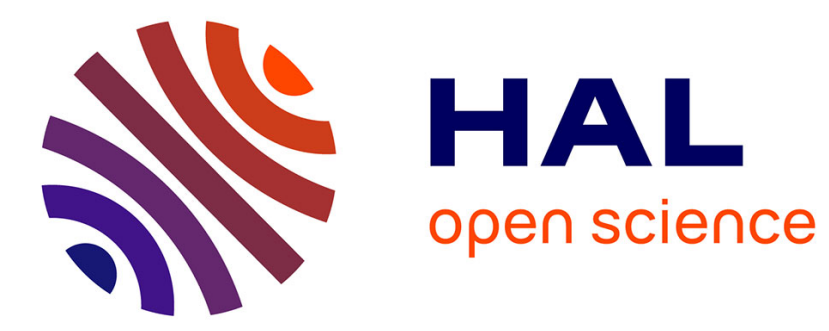

\title{
Le gradient et la transmission intergénérationnelle de la santé pendant l'enfance
}

Bénédicte Apouey, Pierre-Yves Geoffard

\section{To cite this version:}

Bénédicte Apouey, Pierre-Yves Geoffard. Le gradient et la transmission intergénérationnelle de la santé pendant l'enfance. 2015. halshs-01107363

\section{HAL Id: halshs-01107363 \\ https://shs.hal.science/halshs-01107363}

Preprint submitted on 20 Jan 2015

HAL is a multi-disciplinary open access archive for the deposit and dissemination of scientific research documents, whether they are published or not. The documents may come from teaching and research institutions in France or abroad, or from public or private research centers.
L'archive ouverte pluridisciplinaire HAL, est destinée au dépôt et à la diffusion de documents scientifiques de niveau recherche, publiés ou non, émanant des établissements d'enseignement et de recherche français ou étrangers, des laboratoires publics ou privés. 


\section{PARISSCHOOL OF ECONOMICS}

WORKING PAPER N²015 - 02

Le gradient et la transmission intergénérationnelle de la santé pendant l'enfance

Bénédicte H. Apouey

Pierre-Yves Geoffard

JEL Codes: I14, I18

Keywords: inégalités sociales de santé, gradient, transmission

intergénérationnelle de la santé, revenu familial, santé des enfants, santé des parents

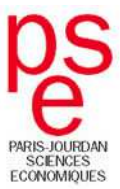




\title{
Le gradient et la transmission intergénérationnelle de la santé pendant l'enfance
}

\author{
Bénédicte H. Apouey* et Pierre-Yves Geoffard**
}

Janvier 2015

* Paris School of Economics - CNRS, Paris, France - benedicte.apouey@ psemail.eu
** Paris School of Economics - CNRS, Paris, France - pierre-yves.geoffard@ psemail.eu

\section{Résumé}

En dépit d'un accès aux soins universel, on observe un gradient de santé dans l'enfance en France, c'est-à-dire une association positive entre le niveau de revenu familial et l'état de santé des enfants.

Cette corrélation peut s'interpréter selon deux chaînes causales qui font toutes deux appel à la santé des parents. Dans une première approche, le revenu familial améliorerait la santé des parents, qui elle-même influencerait positivement la santé des enfants. Dans une seconde approche, la santé des parents aurait un effet à la fois sur le revenu familial et la santé des enfants, créant par là-même une corrélation fallacieuse entre revenu et santé des enfants.

L'effet des politiques publiques sur la santé des enfants sera différent selon les effets à l'œuvre. Si le revenu des parents influence la santé des parents qui elle-même agit sur la santé des enfants, alors une politique qui augmente le revenu de certains ménages entraînera une amélioration de la santé des parents puis des enfants. Mais si la corrélation entre revenu et santé des enfants est fallacieuse, alors une politique de hausse de revenu ne se traduira pas par une amélioration de la santé des enfants. De plus, si la santé des parents a un effet causal sur la santé de leur progéniture, toute mesure permettant d'améliorer la santé des parents est susceptible de bénéficier également aux enfants.

Cet article utilise les données de l'Enquête Santé et Protection Sociale de 1994-2008 pour étudier les relations entre le revenu familial et plusieurs aspects de la santé des enfants et des parents. Nos résultats suggèrent que le revenu a un impact sur la santé digestive et pondérale des enfants, indépendamment de l'effet de la santé des parents. Ce résultat pointe vers le rôle des conditions de vie dans les inégalités sociales de santé pendant l'enfance.

Mots-clés : inégalités sociales de santé, gradient, transmission intergénérationnelle de la santé, revenu familial, santé des enfants, santé des parents.

Codes JEL : I14, I18 


\section{Introduction}

Des études récentes confirment la persistance de disparités sociales d'état de santé et d'espérance de vie en France, en dépit d'un système de soins universel (Apouey, 2010). Ainsi, pour la période allant de 2000 à 2008, l'espérance de vie d'un homme ouvrier âgé de 35 ans est de 40,9 ans en moyenne tandis que celle d'un homme cadre est de 47,2 ans, soit un écart de 6,3 ans. Chez les femmes, des inégalités sociales sont aussi évidentes, même si elles sont moins marquées que chez les hommes (Blanpain, 2011). Des disparités sociales de santé ont également été observées dans la plupart des pays développés (Apouey et Silber, 2013 ;

Marmot et Bobak, 2000). La corrélation entre statut socioéconomique (SSE) et santé, désignée sous les termes de gradient de santé, est assez mal comprise. En effet, dans une population d'adultes, elle peut refléter trois mécanismes, que les études empiriques ont du mal à distinguer : d'abord, un effet causal du SSE sur la santé, si par exemple les individus dont le niveau d'éducation ou de revenu est plus élevé ont accès à des soins de meilleure qualité, et que cela se traduit par une meilleure santé ; ensuite, un effet de la santé sur le SSE, si entre autres les individus en meilleure santé sont en mesure de travailler davantage et de percevoir des salaires plus élevés ; enfin, l'omission de variables, si les modèles ne tiennent pas compte de facteurs inobservés, comme le patrimoine génétique, qui ont un impact à la fois sur le SSE et sur la santé, créant une corrélation entre ces variables sans que pour autant il y ait un quelconque lien de causalité entre elles.

Les travaux qui essaient de mieux comprendre le gradient se sont orientés dans deux directions (Fletcher et Wolfe, 2013). Une première approche consiste à utiliser des chocs exogènes de revenu pour identifier l'effet causal du revenu sur la santé à l'âge adulte (Apouey et Clark, sous presse ; Frijters et al., 2005 ; Kim et Ruhm, 2012 ; Lindahl, 2005). Une seconde approche se concentre sur les enfants des pays développés, parce que l'interprétation du gradient est plus simple pour eux que pour les adultes. En effet, comme les enfants des pays développés ne travaillent pas et ne contribuent pas au revenu familial, leur santé n'a que peu d'effet sur le revenu du ménage. Notre article s'inscrit dans cette seconde approche. Dans ce qui suit, nous passons en revue la littérature relative à cette approche puis nous présentons notre démarche.

Case et al. (2002) examinent le rôle du revenu des parents sur la santé des enfants, à partir de données américaines. Ces auteurs mettent en lumière une corrélation positive entre le revenu familial et la santé subjective des enfants, ainsi qu'une augmentation de cette corrélation avec l'âge des enfants, qu'ils interprètent comme un effet cumulatif du revenu familial sur la santé au cours de l'enfance. A la suite de cette étude, plusieurs articles ont cherché à savoir si ces résultats sont aussi valables dans d'autres pays développés, en particulier des pays dont le système de soins est quasi-universel ou universel. Ainsi, pour le Canada, les deux résultats de Case et al. (2002) sont aussi valides (Currie et Stabile, 2003). En Allemagne, des inégalités sont observées, mais elles n'augmentent pas au cours de l'enfance (Reinhold et Jürges, 2012). En Grande-Bretagne, le gradient de santé est significatif mais son évolution fait débat (Apouey et Geoffard, 2013 ; Case et al., 2008 ; Currie et al., 2007 ; Propper et al., 2007). 
Enfin, en France, le gradient dans l'enfance est significatif et semble se renforcer au cours de l'enfance, comme aux Etats-Unis et au Canada (Apouey et Geoffard, 2014).

Le gradient dans l'enfance s'interprète de deux façons. En premier lieu, il pourrait capturer un effet causal du revenu familial sur la santé des enfants. En effet, un revenu plus élevé pourrait permettre aux enfants d'être mieux nourris, de vivre dans des logements plus sains, d'avoir accès à des soins de meilleure qualité, puis par voie de conséquence d'être en meilleure santé (Propper et al., 2007). En second lieu, le gradient pourrait simplement refléter la présence d'hétérogénéité inobservée dans les modèles, c'est-à-dire l'omission de facteurs influençant à la fois le revenu familial et la santé des enfants.

La santé des parents est susceptible d'entrer en jeu dans chacune de ces deux interprétations du gradient, avec des implications distinctes pour les politiques publiques. Nous nous appuyons sur la figure de l'encadré 1 pour illustrer notre propos.

(i) D'une part, si le gradient reflète un effet causal du revenu sur la santé des enfants, cet effet pourrait transiter par la santé des parents : dans cette perspective, le revenu familial influencerait la santé des parents (flèche (4) dans la figure de l'encadré 1), qui à son tour aurait un impact sur la santé des enfants (flèche (6)). La santé des parents est susceptible d'avoir un impact sur la santé des enfants si les parents en meilleure santé sont plus à mêmes de prendre soin de leurs enfants. Dans cette approche, une politique qui augmenterait le revenu des ménages les plus pauvres engendrerait une amélioration de la santé des enfants de ces ménages.

(ii) D'autre part, si le gradient revenu familial / santé des enfants ne reflète aucunement un effet causal du revenu sur la santé des enfants, mais plutôt l'omission de facteurs, la santé des parents pourrait être l'un de ces facteurs omis. En effet, la santé des parents pourrait avoir un impact à la fois sur le revenu familial (flèche (3)) et la santé des enfants (flèche (6)). Dans ce cas, une politique de redistribution du revenu ne se traduirait pas par une amélioration de la santé des enfants de familles défavorisées. Les interprétations (i) et (ii) du gradient ne sont pas mutuellement exclusives.

En l'absence de riches données longitudinales sur le revenu familial et la santé des parents et des enfants, il n'est pas possible de distinguer les mécanismes (i) et (ii). Cependant, il est tout de même possible d'éclairer le rôle de ces deux mécanismes pris simultanément. Supposons qu'en l'absence de variables de contrôles pour la santé des parents, le gradient de santé dans l'enfance soit fort et significatif. Si lorsque l'on inclut les variables de santé des parents comme contrôles additionnels, le gradient disparaît tandis que la transmission intergénérationnelle de santé apparaît forte, alors on pourra conclure qu'il n'est pas certain qu'une politique de redistribution du revenu permette d'améliorer la santé de certains enfants. En revanche, une politique qui modifierait la santé des parents semblerait prometteuse pour améliorer celle des enfants. 
Dans cet article, nous utilisons les données longitudinales de l'Enquête sur la Santé et la Protection Sociale (ESPS) entre 1994 et 2008 pour étudier l'association entre le revenu familial, la santé des enfants, et la santé des parents en France. Ces données nous permettent d'examiner les liens entre le revenu moyen et différents aspects de la santé des enfants et des parents (santé subjective, morbidité, santé respiratoire et digestive, corpulence). Nous mesurons la santé des parents plusieurs années avant celle des enfants. Nous regardons d'abord s'il existe un gradient entre le revenu familial et la santé des enfants lorsque l'on ne tient pas compte de la santé des parents. Nous nous tournons ensuite vers les mécanismes qui pourraient expliquer ce gradient, en nous concentrant sur le rôle de la santé des parents. La santé des parents pourrait être un canal par lequel le revenu a un effet sur la santé des enfants, mais aussi un facteur omis dans la modèle du gradient. Nous tenons compte de la santé de la mère, mais aussi de celle du père. Cela nous permet de mettre en lumière la transmission intergénérationnelle de la santé qui s'opère pendant l'enfance.

Notre contribution à la recherche sur les déterminants de la santé dans l'enfance est double. En premier lieu, les travaux sur le rôle du SSE familial sur la santé des enfants en France sont très peu nombreux, et notre article enrichit donc les connaissances sur ce sujet (Tursz, 2000). Plus précisément, les quelques études sur données françaises utilisent des échantillons d'enfants de taille restreinte, portent soit sur une région française particulière (et non pas sur l'ensemble du territoire) soit sur un groupe d'âge précis (et non pas sur les enfants de tous âges), et se concentrent sur un petit nombre de variables de santé de l'enfant. Ainsi, chez les enfants de 12 ans du département du Bas-Rhin en 2001, le surpoids est négativement corrélé au revenu imposable et au niveau d'éducation de la mère (Klein-Platat et al., 2003). Pour les adolescents de 11 à 15 ans, vivre dans une famille défavorisée augmente la probabilité d'être en surpoids (Currie et al., 2012). Par contraste, notre recherche utilise un grand échantillon d'enfants de tous âges et de toutes les régions et étudie plusieurs composantes de la santé de l'enfant. Cela nous permet d'offrir une vision plus générale des inégalités sociales de santé dans l'enfance en France. En second lieu, nous contribuons aussi à la recherche sur la transmission intergénérationnelle de la santé, en nous concentrant sur les liens de santé parents-enfant qui émergent pendant l'enfance. La plupart des travaux sur les liens intergénérationnels de santé se focalisent sur la transmission du poids à la naissance (Currie et Moretti, 2005) ou de la santé à l'âge adulte (Ahlburg, 1998), et par opposition, très peu d'analyses considèrent la transmission intergénérationnelle de la santé qui a lieu pendant l'enfance. Ces analyses inspectent l'effet de la santé de la mère sur celle des enfants de moins de sept ans en Grande-Bretagne (Propper et al., 2007), le rôle de la santé subjective des parents sur la santé subjective des enfants aux Etats-Unis (Case et al., 2002), et la transmission intergénérationnelle de la santé pour les enfants de moins de trois ans en Allemagne (Coneus et Spiess, 2012). Nous prolongeons ces études en tenant compte de l'effet de différentes composantes de la santé de la mère et du père, pour un échantillon d'enfants de tous âges, en France.

Notre article met en lumière un gradient positif et significatif pour la santé subjective, digestive, et pondérale de l'enfant. Nous montrons ensuite que le revenu familial est fortement corrélé à la grande majorité de nos variables de santé de la mère et du père. De plus, lorsque 
l'on contrôle pour la santé des parents (mesurée plusieurs années avant celle des enfants), le gradient de santé subjective dans l'enfance disparaît, tandis que le gradient de santé digestive et pondérale reste grand et significatif. La santé subjective et la morbidité des parents ont un impact fort sur tous les aspects de la santé des enfants.

\section{Données}

Les données proviennent de l'Enquête sur la Santé et la Protection Sociale (ESPS) entre 1994 et 2008. Les années exactes de l'enquête sont 1994, 1995, 1996, 1997, 1998, 2000, 2002, 2004, 2006, et 2008. L'ESPS est mise en œuvre par l'Institut de Recherche et Documentation en Economie de la Santé (IRDES) et la Caisse Nationale de l'Assurance Maladie des Travailleurs Salariés (CNAMTS). L'enquête est représentative d'environ 97\% de la population vivant en métropole. Sont exclus les individus vivant dans des ménages collectifs, comme les maisons de retraite et les communautés religieuses, et dans les territoires d'outremer. Les données sont collectées par téléphone, lors d'interview en face-à-face, et par des questionnaires auto-administrés. L'enquête comporte des données détaillées sur l'état de santé, la couverture maladie, et la situation sociale des individus ou des ménages, qui ont été régulièrement utilisées pour étudier les déterminants de la santé pour les adultes (Bricard et Jusot, 2012 ; Cambois et Jusot, 2011 ; Jusot et al., 2013 ; Tubeuf, 2009), mais assez peu pour les enfants (Apouey et Geoffard, 2014).

L'enquête est un panel tournant, qui réinterroge une partie des ménages de l'échantillon dans les vagues suivantes. Au final, les ménages sont souvent (mais pas toujours) interrogés tous les 4 ans ${ }^{1}$. Nous tirons parti de la nature longitudinale des données pour calculer le revenu moyen des ménages (jusqu'à la date de l'enquête), et pour mesurer la santé des parents avant celle des enfants, ce qui permet de limiter la causalité allant de la santé des enfants vers celle des parents. Les données sur les enfants proviennent des vagues 1998 à 2008, tandis que celles sur la santé des parents proviennent des vagues 1994 à 2004.

Les enfants sont définis comme les individus âgés de 0 à 17 ans, qui sont scolarisés ou trop jeunes pour être scolarisés. Cette définition exclut donc les jeunes qui sont actifs. Cette restriction a pour but d'éliminer les mineurs qui ne sont pas dépendants financièrement de leurs parents et qui contribuent au revenu du ménage, pour réduire l'effet de la santé des enfants sur le revenu familial.

La «mère » (respectivement le «père ») est défini(e) comme la femme (resp. l'homme) qui fait partie du même ménage que l'enfant, qui est le «répondant principal » ou le «conjoint du répondant principal » lors de l'enquête, et qui, à la question des liens entre elle (resp. lui) et l'enfant, répond qu'il est son «enfant » dans les vagues 1998 à 2004, ou son « enfant » ou son/sa « beau-fils, belle-fille (au sens d'enfant du conjoint)» dans les vagues 2006 et 2008. Notons que le questionnaire d'ESPS propose la catégorie de réponse « enfant » dans toutes les

\footnotetext{
${ }^{1}$ En 2010, l'échantillon de l'ESPS a été entièrement renouvelé et il n'est donc pas possible d'utiliser cette vague dans une perspective longitudinale.
} 
vagues d'enquête, mais ne propose la catégorie additionnelle «beau-fils, belle-fille » qu'à partir de 2006. En d'autres termes, les données ne permettent pas de distinguer les parents des beaux-parents avant 2006. Dans notre analyse, nous ne faisons jamais la distinction entre parents et beaux-parents. Par abus de langage, nous appelons «mère » la personne qui est en réalité la mère ou la belle-mère de l'enfant, et « père » la personne qui est en réalité le père ou le beau-père de l'enfant. Notre échantillon est constitué des enfants qui vivent avec au moins un de leur parent.

\section{Santé subjective de l'enfant}

La variable de santé subjective de l'enfant provient de la question suivante, posée soit aux parents, soit aux enfants eux-mêmes : «Pouvez-vous [peux-tu] noter, entre 0 et 10, son [ton] état de santé ?» $(0: \text { En très mauvaise santé, } 10 \text { : Excellente santé })^{2}$.

Comme moins d'un pour cent des enfants ont une note de santé comprise entre 1 et 5 , nous regroupons les catégories 1 à 6 . Au final, notre variable de santé subjective possède cinq catégories : 1 (note de 1 à 6), 2 (note de 7), 3 (note de 8), 4 (note de 9), et 5 (note de 10).

\section{Faible morbidité de l'enfant}

Nous utilisons également une variable de faible morbidité, qui est fonction du nombre de maladies de l'enfant. Les parents (ou l'enfant lui-même) déclarent si l'enfant souffre de certain nombre de maladies (voir l'encadré 2). Nous comptons le nombre de maladies de chaque enfant. Notre variable de faible morbidité prend les valeurs suivantes : 1 (4 maladies ou plus), 2 (3 maladies), 3 ( 2 maladies), 4 (1 maladies), et 5 (aucune maladie). Dans notre échantillon, $3,0 \%$ des enfants souffrent de 4 maladies ou plus, et $41,4 \%$ des enfants ne souffrent d'aucune maladie.

De plus, étant donné la fréquence des problèmes respiratoires et digestifs, nous les étudions séparément, à l'aide de variables dichotomiques qui indiquent si l'enfant a une bonne santé respiratoire et digestive. Respectivement $79,9 \%$ et $83,8 \%$ des enfants de notre échantillon sont dans ces cas.

\section{Santé pondérale de l'enfant}

Nous faisons aussi usage d'une variable dichotomique qui indique si l'enfant a une bonne santé pondérale, c'est-à-dire si son indice de masse corporelle (IMC) est normal, par opposition à une situation de sous-poids, de surpoids, ou d'obésité. Cette variable a été construite en utilisant les définitions du sous-poids, surpoids, et obésité de l'International

\footnotetext{
${ }^{2}$ Moins de 5\% des enfants de moins de 10 ans répondent à cette question eux-mêmes (certains de ces cas correspondent certainement à des erreurs dans les réponses dans les données ESPS), contre 12,0\% des adolescents de 12 ans, et 49,8\% des adolescents de 17 ans. La part d'adolescents répondant à la question euxmêmes augmente régulièrement avec l'âge.
} 
Obesity Taskforce (IOTF) de 2012 (Cole et Lobstein, 2012) ${ }^{3}$. La variable de santé pondérale est corrigée pour le sexe et l'âge en mois de l'enfant, et elle est seulement définie pour les enfants de plus de 2 ans. Dans notre échantillon, 69,6\% des enfants de plus de 2 ans sont en bonne santé pondérale.

\section{Santé des parents}

ESPS interroge chacun des parents sur son propre état de santé. Pour chacun d'eux, nous utilisons des variables de santé similaires à celles des enfants, ce qui nous permet d'avoir un éventail d'indicateurs de santé assez grand ${ }^{4}$. Dans nos modèles économétriques, toutes les variables de santé des parents sont retardées, c'est-à-dire mesurées entre 3 et 5 ans avant les autres variables, et notamment la santé des enfants. Cela permet de limiter la causalité inverse allant de la santé des enfants vers celle des parents.

\section{Remarques sur les variables de santé}

Les variables de santé des enfants proviennent d'un « questionnaire santé » qui est rempli soit par les parents soit par les enfants. Quant aux variables de santé des parents, elles sont rapportées par les parents eux-mêmes. Ainsi dans nos données, les variables d'état de santé sont toujours rapportées par les parents ou les enfants, et jamais par un professionnel de santé. A ce titre nos variables de santé sont subjectives et non pas objectives.

Dans notre échantillon total d'enfants de tous âges, ce sont généralement les parents qui répondent au questionnaire de santé des enfants. Cela est susceptible d'engendrer des biais de déclaration, dans le sens où l'état de santé des parents pourrait avoir un impact sur la façon dont ils rapportent l'état de santé de leur progéniture. Ainsi, des parents déprimés pourraient sous-estimer l'état de santé de leurs enfants. En outre, des parents en mauvaise santé qui sont régulièrement en contact avec le milieu médical pourraient porter un jugement plus objectif sur la santé de leurs enfants que les autres parents. Dans nos modèles, la santé des parents est mesurée 3 à 5 ans avant celle des enfants, ce qui diminue certainement les biais de déclaration, mais ne les élimine pas pour autant. La corrélation entre la santé des parents et celle des enfants que nous observons dans nos données ne reflète pas seulement la transmission intergénérationnelle de la santé, mais aussi ces biais de déclaration. Nous gardons cette limitation à l'esprit dans ce qui suit.

\section{Revenu du ménage}

La variable de statut socio-économique du ménage est le logarithme du revenu mensuel moyen. Plus précisément, les données originales contiennent pour chaque ménage un revenu

\footnotetext{
${ }^{3}$ Voir http://www.worldobesity.org/aboutobesity/child-obesity/newchildcutoffs/ également.

${ }^{4}$ La santé pondérale des parents indique que leur corpulence est normale (IMC supérieur ou égal à 18,5 et strictement inférieur à 25), par opposition à un état de maigreur (IMC strictement inférieur à 18,5), de surpoids, ou d'obésité (IMC supérieur ou égal à 25). Voir la classification internationale de la corpulence des adultes sur la page de l'Organisation Mondiale de la Santé : http://apps.who.int/bmi/index.jsp?introPage=intro_3.html
} 
annuel exact ou en tranches, selon les vagues, et selon la préférence du répondant pour indiquer son revenu exact ou non. Nous utilisons le revenu exact lorsqu'il est disponible et le milieu de la tranche sinon. Ce type d'imputation du revenu a déjà été utilisé dans la littérature antérieure (Currie et al., 2007). Des méthodes alternatives d'imputation du revenu sont disponibles $^{5}$. Le revenu est corrigé de l'inflation grâce à un indice des prix ${ }^{6}$. Nous calculons le revenu moyen du ménage jusqu'à la date de l'enquête, et pour tenir compte de la non-linéarité de la relation entre revenu et santé, nous utilisons le logarithme du revenu moyen (Case et al., 2002).

\section{Variables de contrôle}

Les modèles économétriques incluent un certain nombre de variables de contrôle, qui sont proches de celles utilisées dans les travaux antérieurs sur les inégalités de santé dans l'enfance (Case et al., 2002 ; Currie et al., 2007 ; Reinhold et Jürges, 2012). Une première liste de variables de contrôle, « contrôles 1 », comprend des variables indicatrices pour le type de famille dans laquelle vit l'enfant (soit une famille avec sa mère et son père (cette variable est utilisée comme référence), soit une famille monoparentale avec sa mère, soit une famille monoparentale avec son père), une variable indicatrice pour le sexe de l'enfant, des variables indicatrices pour l'âge de l'enfant (les enfants de 10 ans servent de référence), l'âge de la mère et du père (interagis avec leur présence dans la ménage), le logarithme de la taille du ménage, des variables indicatrices pour l'identité du répondant aux questions de santé de l'enfant (soit le répondant est l'un des deux parents (référence), soit c'est l'enfant lui-même, soit c'est un répondant non identifié dans les données), et des variables dichotomiques pour l'année de l'enquête. Une seconde liste de contrôles, « contrôles 2 », contient en plus des « contrôles 1 » le niveau d'éducation ${ }^{7}$ et le statut d'emploi de la mère (interagis avec sa présence dans le ménage).

Le tableau 1 présente les statistiques descriptives pour les variables d'intérêt et certaines variables de contrôle. Au sein de notre échantillon (de 11889 enfants), 48,0\% des enfants sont des filles, l'âge moyen est d'un peu moins de 9 ans, et 7,4\% des enfants vivent dans un ménage monoparental (avec leur mère ou leur père).

\footnotetext{
${ }^{5}$ Une première alternative à notre principale méthode d'imputation du revenu consiste à utiliser une régression par intervalle. Cette technique consiste à régresser les bornes inférieure et supérieure de chaque tranche de revenu sur un certain nombre de variables qui expliquent le revenu du ménage, puis de prédire ce revenu. Lorsque nous avons mis en œuvre cette méthode, la qualité de l'imputation n'était pas assez bonne. Une autre alternative consiste à utiliser le milieu empirique de la tranche, qui est lui-même calculé à partir des revenus exacts. La moyenne du logarithme du revenu obtenu avec cette méthode est très proche de celle obtenue avec notre méthode. En outre, les estimations du gradient sont également très proches. Ces résultats sont disponibles auprès des auteurs.

${ }^{6}$ Nous corrigeons de l'érosion monétaire en utilisant le convertisseur suivant : http://www.insee.fr/fr/themes/calcul-pouvoir-achat.asp

${ }^{7}$ Le niveau d'éducation de la mère est codé en trois catégories : niveau d'éducation faible, moyen, et élevé. Le niveau d'éducation faible correspond à un niveau inférieur à la classe de sixième du collège. Cette catégorie regroupe les personnes qui n'ont pas été scolarisées, qui sont seulement allées à l'école maternelle ou primaire, ou qui ont un certificat d'études primaire. Le niveau d'éducation moyen correspond à un niveau inférieur à la classe de troisième du collège, à un CAP ou à un BEP. Enfin, le niveau d'éducation élevé correspond à un niveau d'étude supérieur à la classe de seconde du lycée.
} 
Tableau 1. Statistiques descriptives

\begin{tabular}{|c|c|c|c|}
\hline Variables & Vagues & Observations & Moyennes (écarts-types) \\
\hline \multicolumn{4}{|l|}{ Variables de santé de l'enfant } \\
\hline (Bonne) Santé subjective - Enfant & $1998-2008$ & $11889^{\#}$ & \\
\hline 1 (note entre 1 et 6 ) & & & 0,0177 \\
\hline 2 (note de 7$)$ & & & 0,0284 \\
\hline 3 (note de 8 ) & & & 0,1288 \\
\hline 4 (note de 9) & & & 0,2309 \\
\hline $5($ note de 10$)$ & & & 0,5939 \\
\hline Faible morbidité - Enfant & $1998-2008$ & $12343^{\#}$ & \\
\hline 1 (4 maladies ou plus) & & & 0,0304 \\
\hline 2 (3 maladies) & & & 0,0611 \\
\hline 3 (2 maladies) & & & 0,1639 \\
\hline 4 (1 maladie) & & & 0,3302 \\
\hline 5 (aucune maladie) & & & 0,4141 \\
\hline (Bonne) Santé respiratoire - Enfant & $1998-2008$ & $12343^{\#}$ & 0,7998 \\
\hline (Bonne) Santé digestive - Enfant & $1998-2008$ & $12343^{\#}$ & 0,8383 \\
\hline (Bonne) Santé pondérale - Enfant & $1998-2008$ & $10001^{\#}$ & 0,6969 \\
\hline \multicolumn{4}{|l|}{ Revenu familial } \\
\hline Log(revenu moyen) & 1998-2008 & $11889^{\#}$ & $7,8819(0,4022)$ \\
\hline \multicolumn{4}{|l|}{ Variables retardées de santé de la mère } \\
\hline (Bonne) Santé subjective - Mère & 1994-2004 & $6448^{\# \#}$ & \\
\hline 1 (note entre 1 et 6 ) (référence) & & & 0,0780 \\
\hline 2 (note de 7 ) & & & 0,1073 \\
\hline 3 (note de 8 ) & & & 0,2921 \\
\hline 4 (note de 9) & & & 0,2392 \\
\hline 5 (note de 10$)$ & & & 0,2831 \\
\hline Faible morbidité - Mère & 1994-2004 & $6547^{\# \#}$ & \\
\hline 1 (4 maladies ou plus) (référence) & & & 0,2566 \\
\hline 2 (3 maladies) & & & 0,2124 \\
\hline 3 (2 maladies) & & & 0,2640 \\
\hline 4 (1 maladie) & & & 0,2080 \\
\hline 5 (aucune maladie) & & & 0,0588 \\
\hline (Bonne) Santé respiratoire-Mère & $1994-2004$ & $6547^{\# \#}$ & 0,8212 \\
\hline (Bonne) Santé digestive-Mère & $1994-2004$ & $6547^{\# \#}$ & 0,3108 \\
\hline (Bonne) Santé pondérale-Mère & $1994-2004$ & $6451^{\# \#}$ & 0,6926 \\
\hline \multicolumn{4}{|l|}{ Variables retardées de santé du père } \\
\hline (Bonne) Santé subjective-Père & 1994-2004 & $6071^{\# \#}$ & \\
\hline 1 (note entre 1 et 6 ) (référence) & & & 0,0655 \\
\hline 2 (note de 7 ) & & & 0,1073 \\
\hline 3 (note de 8 ) & & & 0,2763 \\
\hline 4 (note de 9) & & & 0,2409 \\
\hline $5($ note de 10$)$ & & & 0,3096 \\
\hline Faible morbidité-Père & 1994-2004 & $6232^{\# \#}$ & \\
\hline
\end{tabular}




\begin{tabular}{|c|c|c|c|}
\hline 1 (4 maladies ou plus) & & & 0,1577 \\
\hline 2 (3 maladies) & & & 0,1943 \\
\hline 3 (2 maladies) & & & 0,3026 \\
\hline 4 (1 maladie) & & & 0,2759 \\
\hline 5 (aucune maladie) & & & 0,0693 \\
\hline (Bonne) Santé respiratoire - Père & 1994-2004 & $6232^{\# \#}$ & 0,8621 \\
\hline (Bonne) Santé digestive - Père & 1994-2004 & $6232^{\# \#}$ & 0,3263 \\
\hline (Bonne) Santé pondérale-Père & 1994-2004 & $6160^{\# \#}$ & 0,5547 \\
\hline Variables de contrôle & $1998-2008$ & $11889^{\#}$ & \\
\hline Famille avec mère et père (référence) & & & 0,9247 \\
\hline Famille monoparentale avec mère & & & 0,0660 \\
\hline Famille monoparentale avec père & & & 0,0089 \\
\hline Edu faible (interagie avec la présence) - Mère (référence) & & & 0,4430 \\
\hline Edu moyenne (interagie avec la présence) - Mère & & & 0,1909 \\
\hline Edu élevée (interagie avec la présence) - Mère & & & 0,3565 \\
\hline En emploi (interagi avec la présence) - Mère & & & 0,7521 \\
\hline Fille & & & 0,4807 \\
\hline Moins d'1 an & & & 0,0221 \\
\hline 1 an & & & 0,0458 \\
\hline 2 ans & & & 0,0515 \\
\hline 3 ans & & & 0,0502 \\
\hline 4 ans & & & 0,0587 \\
\hline 5 ans & & & 0,0608 \\
\hline 6 ans & & & 0,0578 \\
\hline 7 ans & & & 0,0602 \\
\hline 8 ans & & & 0,0603 \\
\hline 9 ans & & & 0,0627 \\
\hline 10 ans (référence) & & & 0.0627 \\
\hline 11 ans & & & 0,0571 \\
\hline 12 ans & & & 0,0607 \\
\hline 13 ans & & & 0,0618 \\
\hline 14 ans & & & 0,0579 \\
\hline 15 ans & & & 0,0580 \\
\hline 16 ans & & & 0,0610 \\
\hline 17 ans & & & 0,0545 \\
\hline Age (interagi avec la présence) - Mère & & & $37,6799(7,0000)$ \\
\hline Age (interagi avec la présence) - Père & & & $37,6642(11,9129)$ \\
\hline Log(taille du ménage) & & & $1,4248(0,2355)$ \\
\hline Parents déclarent la santé de l'enfant (référence) & & & 0,8241 \\
\hline Enfant déclare sa santé & & & 0,1417 \\
\hline Inconnu déclare la santé de l'enfant & & & 0,0344 \\
\hline
\end{tabular}

Lecture : les écart-types des variables continues sont indiqués entre parenthèses.

Champ : ménages ordinaires vivant en France métropolitaine.

Source : vagues 1994-2008 de l'enquête Santé et Protection Sociale (ESPS) de l'IRDES, calcul des auteurs.

\# Cet échantillon est utilisé dans le tableau 2, spécification A. ${ }^{\# \#}$ Cet échantillon est utilisé dans le tableau 3 et dans le tableau 4, colonne (1).

\section{Modèles pour le gradient et la transmission intergénérationnelle de la santé}

L'objectif de cet article est de mesurer le gradient dans l'enfance pour un certain nombre de variables de santé de l'enfant, d'éclairer le rôle de la santé des parents dans ce gradient, et de quantifier la transmission intergénérationnelle de la santé qui s'opère pendant l'enfance. Nous 
nous appuyons sur trois modèles sous forme réduite, qui nous permettent de mesurer des associations entre nos variables d'intérêt. Nous faisons le choix de ne pas nous intéresser aux relations structurelles entre ces variables et nous interprétons nos résultats en termes de corrélation et non pas de causalité.

Le premier modèle quantifie le gradient revenu / santé de l'enfant, lorsque l'on ne tient pas compte de la santé des parents. Dans le cadre de la fonction de production de santé de l'enfant, l'état de santé de l'enfant est fonction du revenu familial et d'un certain nombre d'autres variables individuelles et familiales (Grossman, 2000). La forme réduite correspondante s'écrit de la façon suivante :

$S^{e}=\alpha_{1}^{I}+\alpha_{2}^{I} X+\beta_{1}^{I} Y+\varepsilon^{I}$

Où $S^{e}$ est une variable de santé de l'enfant, $Y$ est le logarithme du revenu des parents, $X$ représente un vecteur de variables individuelles et familiales de contrôle, et $\varepsilon^{I}$ est un terme résiduel. On s'attend à ce que pour certaines variables de santé de l'enfant, le coefficient du revenu $\beta_{1}^{I}$ soit positif et significatif, ce qui signifierait que les enfants issus de milieux plus aisés sont en meilleure santé. Le modèle (I) est le modèle standard d'évaluation du gradient dans l'enfance (Case et al., 2002).

Après avoir mesuré le gradient, nous nous tournons vers les mécanismes sous-jacents, en nous intéressant à la santé des parents. La santé des parents pourrait jouer un rôle dans deux mécanismes expliquant le gradient pour deux raisons. En premier lieu, si le revenu a un effet causal sur la santé des enfants, cet impact pourrait s'effectuer à travers la santé des parents : le revenu influencerait alors la santé des parents qui aurait un effet sur la santé des enfants. En second lieu, la santé des parents pourrait être un facteur omis dans le modèle (I). En effet, la santé des parents est susceptible d'être associée à la fois au revenu familial et à la santé des parents, créant par là-même une corrélation fallacieuse entre revenu et santé des enfants. Dans cette perspective, le gradient mis en évidence ne reflèterait nullement un effet causal du revenu sur la santé des enfants.

Pour que la santé des parents soit un mécanisme expliquant le gradient dans le modèle (I), il est impératif qu'elle soit corrélée au revenu familial, quel que soit le mécanisme retenu (Propper et al., 2007). Nous commençons donc par examiner si cela est vrai, en régressant les différentes variables de santé des parents sur le logarithme du revenu familial :

$S^{p}=\alpha^{I I}+\gamma^{I I} Y+\varepsilon^{I I}$

Où $S^{p}$ est une variable de santé d'un parent. Nous estimons ce modèle en l'absence de contrôles, dans la mesure où nous souhaitons simplement vérifier la pertinence de notre hypothèse. 
Finalement, nous incluons les variables de santé des parents dans le modèle (I). Cela nous permet d'une part de vérifier si le gradient est robuste à l'inclusion de ces contrôles, et d'autre part d'évaluer le niveau de transmission de santé entre générations (Case et al., 2002 ; Propper et al., 2007). Pour cela, nous estimons le modèle suivant :

$S^{e}=\alpha_{1}^{I I I}+\alpha_{2}^{I I I} X+\beta_{1}^{I I I} Y+\beta_{2}^{I I I} S^{p}+\varepsilon^{I I I}$

Où $S^{p}$ désigne la santé de la mère ou du père. Toutes les variables, en particulier la santé des enfants, sont mesurées l'année de l'enquête, à la date $t$, sauf la santé des parents qui est mesurée trois à cinq ans auparavant, en $t-3, t-4$ ou $t-5$, afin de limiter tout effet causal allant de la santé des enfants vers celle des parents. Dans ce modèle, $\beta_{1}^{I I I}$ nous informe de la robustesse du gradient. Ainsi, si $\beta_{1}^{I}$ est significatif dans le modèle (I) et $\beta_{1}^{I I I}$ reste significatif dans le modèle (III) lorsque l'on tient compte de la santé des parents, alors le revenu exerce un rôle sur la santé des enfants indépendamment de la santé des parents. Au contraire, si $\beta_{1}^{I}$ est significatif mais $\beta_{1}^{I I I}$ n'est plus significatif, alors la santé des parents est un mécanisme sousjacent au gradient. En d'autres termes, dans le modèle (I), la santé des parents est soit un facteur intermédiaire dans la causalité allant du revenu vers la santé des enfants, soit une variable omise. $\beta_{2}^{I I I}$ quantifie la force des liens intergénérationnels de santé.

L'encadré 1 résume les relations entre le revenu familial et la santé des enfants et des parents.

\section{Le gradient revenu familial / santé dans l'enfance}

Nous commençons par quantifier le gradient de santé dans l'enfance, pour nos cinq variables de santé de l'enfant, à l'aide du modèle standard (I). Ce modèle est estimé à l'aide d'un probit simple ou ordonné, selon que la variable expliquée de santé de l'enfant possède deux catégories ou plus. Le tableau 2 présente les résultats. Dans la spécification A, les modèles incluent les « contrôles 1 » comme variables de contrôle. La colonne (1) contient les résultats lorsque la variable dépendante est la santé subjective de l'enfant, et montre une corrélation positive et significative entre revenu moyen et santé subjective. En termes d'effet marginal, le coefficient du revenu implique que lorsque le revenu familial passe du $25^{\text {ème }}$ au $75^{\text {ème }}$ percentile, la probabilité que l'enfant ait le score de santé subjective le plus élevé augmente de 3,7 points de pourcentage, passant de $57,8 \%$ à $61,5 \%$. De même, les colonnes (5) à (6) mettent en évidence une association positive et significative entre le revenu et la santé digestive et pondérale. En revanche, les associations entre le revenu et la faible morbidité d'une part, et entre le revenu et la santé respiratoire d'autre part, ne sont pas significative.

La spécification B présente l'association entre le revenu moyen et la santé lorsque les « contrôles 2 » sont inclus. On observe à nouveau une corrélation positive et significative entre le revenu moyen et la santé subjective, digestive, et pondérale. En termes d'effets marginaux, une hausse du revenu moyen du $25^{\text {ème }}$ percentile au $75^{\text {ème }}$ percentile va de pair avec une augmentation de la probabilité que l'enfant ait le score de santé subjective le plus élevé de 3,8 points de pourcentage (de $57,8 \%$ à $61,6 \%$ ), qu'il ait une bonne santé digestive de 
1,6 point de pourcentage (de $85,8 \%$ à $87,4 \%$ ), et qu'il ait une corpulence normale de 3,0 points de pourcentage (de $68,6 \%$ à $71,6 \%$ ).

Le bas du tableau 2 montre les coefficients des variables de contrôle. Les enfants vivant dans une famille monoparentale avec leur mère sont en meilleure santé subjective et en moins bonne santé digestive et pondérale que les enfants vivant dans une famille avec leur mère et leur père. Les enfants vivant dans une famille monoparentale avec leur père ne semblent pas avoir un état de santé significativement différent des enfants vivant dans une famille avec leur mère et leur père, toutes choses égales par ailleurs. En général, le niveau d'éducation et le statut d'emploi des mères ne sont pas significativement associés à la santé des enfants. Pour cette raison, nous utilisons seulement les « contrôles 1 » dans le reste de l'article. Les filles sont en meilleure santé respiratoire que les garçons, toutes choses égales par ailleurs. La santé subjective des plus de 11 ans est significativement moins bonne que celle des enfants de 10 ans, et une dégradation de la santé subjective continue se dessine entre 10 et 17 ans. De plus, on observe une dégradation progressive de la morbidité au cours de l'enfance. Si la taille du ménage est positivement et significativement associée à la santé subjective, à la faible morbidité, et à la santé respiratoire, elle est en revanche négativement corrélée à la santé digestive. Les enfants qui répondent eux-mêmes aux questions portant sur leur propre santé déclarent une moins bonne santé subjective et respiratoire et une plus forte morbidité. 
Tableau 2. Le gradient de revenu moyen pour différents aspects de la santé des enfants

\begin{tabular}{lccccc}
\hline & $(1)$ & $(2)$ & $(3)$ & $(4)$ & $(5)$ \\
Variable expliquée & Santé & Faible & Santé & Santé & Santé \\
& Subjective & morbidité & respiratoire & digestive & pondérale \\
& Enfant & Enfant & Enfant & Enfant & Enfant \\
Méthode & à la date $t$ & à la date $t$ & à la date $t$ & à la date $t$ & à la date $t$ \\
\hline
\end{tabular}

Spécification A. En incluant les « contrôles 1 »

Log(revenu moyen)

Observations

0,0410
$(0,0288)$
12343

$(0,0319)$

11889

0,0587
$(0,0372)$
12343

Spécification B. En incluant les « contrôles 2 »

Log(revenu moyen)

Variables de contrôle à la date $t$

Famille monoparentale avec mère

Famille monoparentale avec père

Edu moyenne - Mère

Edu élevée - Mère

En emploi - Mère

Fille

Moins d'1 an

1 an

2 ans

3 ans

4 ans

5 ans

6 ans

7 ans

8 ans

9 ans

11 ans

12 ans

13 ans

14 ans

\section{$0,2132 * * *$} $(0,0375)$

\section{0,0412} $(0,0438)$
$0,1303 * * *$
$(0,0463)$

$0,1472 * * *$

$(0,0336)$

$(0,0448)$

$\begin{array}{ccccc}0,2831 * * & -0,1010 & 0,0812 & -0,4087 * * & -0,2717 * \\ (0,1290) & (0,1195) & (0,1484) & (0,1650) & (0,1549) \\ 0,0730 & 0,2340 & 0,2410 & 0,1785 & 0,1551 \\ (0,1760) & (0,1663) & (0,2064) & (0,2263) & (0,2174) \\ -0,0032 & -0,0006 & 0,0112 & 0,0359 & 0,0277 \\ (0,0332) & (0,0302) & (0,0385) & (0,0403) & (0,0387) \\ -0,0439 & -0,0244 & 0,0146 & 0,0674 * & 0,0357 \\ (0,0308) & (0,0280) & (0,0362) & (0,0394) & (0,0376) \\ 0,0035 & 0,0065 & 0,0224 & -0,0527 & -0,0026 \\ (0,0291) & (0,0264) & (0,0341) & (0,0374) & (0,0350) \\ -0,0288 & -0,0303 & 0,1122 * * * & -0,0509 * & -0,0463 \\ (0,0235) & (0,0214) & (0,0278) & (0,0298) & (0,0285) \\ 0,1965 * & 0,7880 * * * & 0,3267 * * * & 0,2313 * & \\ (0,1007) & (0,0846) & (0,1184) & (0,1246) & \\ 0,0678 & 0,6353 * * * & -0,0798 & 0,3730 * * * & \\ (0,0720) & (0,0666) & (0,0851) & (0,1026) & \\ -0,0939 & 0,4693 * * * & -0,2344 * * * & 0,8860 * * * & -0,3545 * * * \\ (0,0692) & (0,0632) & (0,0809) & (0,1168) & (0,0914) \\ -0,1715 * * & 0,3653 * * * & -0,2137 * * * & 0,7607 * * * & -0,3528 * * * \\ (0,0710) & (0,0640) & (0,0812) & (0,1093) & (0,0794) \\ -0,0738 & 0,2831 * * * & -0,2802 * * * & 0,6125 * * * & -0,2285 * * * \\ (0,0661) & (0,0605) & (0,0764) & (0,1030) & (0,0749) \\ -0,0522 & 0,2400 * * * & -0,1898 * * & 0,6064 * * * & -0,1496 * * \\ (0,0633) & (0,0573) & (0,0754) & (0,0962) & (0,0723) \\ -0,0837 & 0,1493 * * & -0,1385 * & 0,3112 * * * & -0,1335 * \\ (0,0656) & (0,0592) & (0,0767) & (0,0874) & (0,0741) \\ -0,0665 & 0,1439 * * & -0,1149 & 0,1239 & 0,0650 \\ (0,0654) & (0,0565) & (0,0770) & (0,0835) & (0,0746) \\ 0,0523 & 0,0944 * & 0,0771 & 0,0893 & -0,0380 \\ (0,0659) & (0,0566) & (0,0788) & (0,0819) & (0,0739) \\ -0,0897 & 0,0300 & 0,1390 * & -0,1507 * & 0,0963 \\ (0,0644) & (0,0563) & (0,0795) & (0,0781) & (0,0737) \\ -0,1429 * * & -0,0513 & 0,1284 & -0,1911 * * & 0,1237 \\ (0,0658) & (0,0568) & (0,0816) & (0,0797) & (0,0761) \\ -0,1399 * * & -0,0598 & 0,0310 & -0,1450 * & 0,1639 * * \\ (0,0634) & (0,0563) & (0,0779) & (0,0797) & (0,0740) \\ -0,1754 * * * & -0,1039 * & 0,0931 & -0,1991 * * & 0,1609 * * \\ (0,0618) & (0,0557) & (0,0765) & (0,0784) & (0,0734) \\ -0,2269 * * * & -0,1222 * * & 0,1029 & -0,2385 * * * & 0,2784 * * * \\ & & & & \end{array}$




\begin{tabular}{lccccc} 
& $(0,0646)$ & $(0,0583)$ & $(0,0814)$ & $(0,0797)$ & $(0,0785)$ \\
15 ans & $-0,3160 * * *$ & $-0,2067 * * *$ & $0,1351 *$ & $-0,2438 * * *$ & $0,2976 * * *$ \\
& $(0,0654)$ & $(0,0588)$ & $(0,0818)$ & $(0,0810)$ & $(0,0795)$ \\
16 ans & $-0,400)^{* * *}$ & $-0,2730 * * *$ & 0,1163 & $-0,2604 * * *$ & $0,2187 * * *$ \\
& $(0,0639)$ & $(0,0582)$ & $(0,0810)$ & $(0,0802)$ & $(0,0783)$ \\
17 ans & $-0,5044 * * *$ & $-0,3482 * * *$ & $-0,0054$ & $-0,3235 * * *$ & $0,3094 * * *$ \\
& $(0,0656)$ & $(0,0621)$ & $(0,0834)$ & $(0,0827)$ & $(0,0823)$ \\
Age - Mère & $-0,0008$ & 0,0026 & $-0,0023$ & $0,0082 * *$ & 0,0032 \\
& $(0,0032)$ & $(0,0030)$ & $(0,0037)$ & $(0,0041)$ & $(0,0040)$ \\
Age - Père & $0,0058 * *$ & $-0,0005$ & 0,0017 & $-0,0043$ & $-0,0061 *$ \\
& $(0,0028)$ & $(0,0026)$ & $(0,0032)$ & $(0,0036)$ & $(0,0034)$ \\
Log(taille du ménage) & $0,2532 * * *$ & $0,2546 * * *$ & $0,3261 * * *$ & $-0,1247 *$ & $-0,0555$ \\
& $(0,0587)$ & $(0,0535)$ & $(0,0650)$ & $(0,0709)$ & $(0,0705)$ \\
Enfant déclare sa santé & $-0,1441 * * *$ & $-0,1471 * * *$ & $-0,1556 * * *$ & $-0,0117$ & 0,0613 \\
& $(0,0337)$ & $(0,0320)$ & $(0,0422)$ & $(0,0411)$ & $(0,0426)$ \\
Inconnu déclare la santé & $-0,1228 *$ & $0,1817 * * *$ & $-0,0008$ & 0,1001 & $-0,0573$ \\
de l'enfant & $(0,0670)$ & $(0,0544)$ & $(0,0705)$ & $(0,0741)$ & $(0,0815)$ \\
& & & & & \\
Observations & 11741 & 12186 & 12186 & 12186 & 9877 \\
\hline
\end{tabular}

Lecture : Les échantillons comprennent des enfants vivant dans une famille avec leur mère et leur père, dans une famille monoparentale avec leur mère, ou dans une famille monoparentale avec leur père. Dans la spécification A, les « contrôles 1 » sont inclus, c'est-à-dire : le type de ménage, le sexe de l'enfant, des variables indicatrices pour l'âge de l'enfant, l'âge de la mère et du père interagi avec leur présence dans la ménage, le logarithme de la taille du ménage, l'identité du répondant aux questions de santé de l'enfant, et l'année de l'enquête. Dans la spécification $\mathrm{B}$, les «contrôles 2 » sont inclus, c'est-à-dire : les « contrôles 1 » et le niveau d'éducation et le statut d'emploi de la mère interagis avec sa présence dans le ménage. Les catégories de référence sont : les familles avec la mère et le père, le niveau d'éducation faible de la mère, les enfants de 10 ans, les parents déclarant la santé de l'enfant.

Les écarts-types sont donnés entre parenthèses.

Seuils de significativité du test de rejet de l'hypothèse de nullité du coefficient : *** 1\%,**5\%,*10\%. Champ : ménages ordinaires vivant en France métropolitaine.

Source : vagues 1998-2008 de l'enquête Santé et Protection Sociale (ESPS) de l'IRDES, calculs des auteurs.

\section{L'effet du revenu et de la santé des parents}

Nous nous penchons maintenant sur le rôle de la santé des parents. Pour qu'elle joue un rôle dans le gradient, il est nécessaire qu'elle soit corrélée au revenu familial. Nous commençons par vérifier ce point en estimant le modèle (II), sans inclure de variables de contrôles, à l'aide de modèles probit simples ou ordonnés. Les résultats sont présentés dans le tableau 3.

En général, le revenu moyen est positivement et significativement associé à la santé des parents. En particulier, il est positivement corrélé à la santé subjective, digestive, et pondérale, et à la faible morbidité de la mère, ainsi qu'à la santé subjective et respiratoire du père.

En revanche, le revenu moyen est négativement et significativement associé à la faible morbidité du père. Nous avons examiné ce point plus en détail et trouvé que le revenu courant est aussi négativement associé à la faible morbidité du père, aussi bien dans l'échantillon utilisé dans le tableau 3 (qui est constitué des parents d'enfants suivis à travers le temps) que dans l'échantillon total de l'ESPS. Une interprétation de cette corrélation est que les hommes aux revenus plus faibles seraient moins susceptibles d'aller chez le médecin, et donc seraient moins informés et sous-déclareraient certaines maladies. 
De façon générale, les résultats du tableau 3 confirment cependant que la santé de parents est susceptible d'être un mécanisme expliquant le gradient dans l'enfance, et soulignent l'intérêt d'étudier plusieurs composantes de la santé des parents et d'examiner le rôle de la santé du père en plus de celle de la mère.

Tableau 3. La corrélation entre le revenu moyen et la santé des parents

\begin{tabular}{|c|c|c|c|c|c|}
\hline Variable expliquée & $\begin{array}{c}(1) \\
\text { Santé } \\
\text { subjective } \\
\text { Mère } \\
\end{array}$ & $\begin{array}{c}(2) \\
\text { Faible } \\
\text { Morbidité } \\
\text { Mère }\end{array}$ & $\begin{array}{c}(3) \\
\text { Santé } \\
\text { respiratoire } \\
\text { Mère } \\
\end{array}$ & $\begin{array}{c}\text { (4) } \\
\text { Santé } \\
\text { digestive } \\
\text { Mère }\end{array}$ & $\begin{array}{c}(5) \\
\text { Santé } \\
\text { pondérale } \\
\text { Mère } \\
\end{array}$ \\
\hline Méthode & Probit ordonné & Probit ordonné & Probit & Probit & Probit \\
\hline $\begin{array}{l}\text { Log(revenu moyen) } \\
\text { Observations }\end{array}$ & $\begin{array}{c}0,3332 * * * \\
(0,0385) \\
6448\end{array}$ & $\begin{array}{c}0,0636^{*} \\
(0,0382) \\
6547\end{array}$ & $\begin{array}{c}0,0531 \\
(0,0499) \\
6547\end{array}$ & $\begin{array}{c}0,1648 * * * \\
(0,0419) \\
6547\end{array}$ & $\begin{array}{c}0,4381 * * * \\
(0,0497) \\
6451\end{array}$ \\
\hline Variable expliquée & $\begin{array}{c}\text { (6) } \\
\text { Santé } \\
\text { subjective } \\
\text { Père }\end{array}$ & $\begin{array}{c}(7) \\
\text { Faible } \\
\text { Morbidité } \\
\text { Père }\end{array}$ & $\begin{array}{l}(8) \\
\text { Santé } \\
\text { respiratoire } \\
\text { Père }\end{array}$ & $\begin{array}{c}(9) \\
\text { Santé } \\
\text { digestive } \\
\text { Père }\end{array}$ & $\begin{array}{c}(10) \\
\text { Santé } \\
\text { pondérale } \\
\text { Père }\end{array}$ \\
\hline Méthode & Probit ordonné & Probit ordonné & Probit & Probit & Probit \\
\hline $\begin{array}{l}\text { Log(revenu moyen) } \\
\text { Observations }\end{array}$ & $\begin{array}{c}0,2630 * * * \\
(0,0421) \\
6071\end{array}$ & $\begin{array}{c}-0,2061 * * * \\
(0,0403) \\
6232\end{array}$ & $\begin{array}{c}0,1070 * \\
(0,0581) \\
6232\end{array}$ & $\begin{array}{c}0,0550 \\
(0,0437) \\
6232\end{array}$ & $\begin{array}{c}0,0811 \\
(0,0493) \\
6160\end{array}$ \\
\hline
\end{tabular}

Lecture : les écarts-types sont donnés entre parenthèses.

Seuils de significativité du test de rejet de l'hypothèse de nullité du coefficient : *** $1 \%$, ** 5\%,* $10 \%$.

Champ : ménages ordinaires vivant en France métropolitaine.

Source : vagues 1994-2008 de l'enquête Santé et Protection Sociale (ESPS) de l'IRDES, calculs des auteurs.

Nous poursuivons l'analyse en estimant le modèle (III) du gradient, qui inclut les contrôles pour la santé des parents. Les résultats sont présentés dans le tableau 4. Dans les différentes spécifications de ce tableau, nous étudions séparément l'impact des différentes variables de santé des parents. Il n'est pas possible d'inclure toutes les variables de santé des parents dans le même modèle, car elles sont parfois fortement corrélées entre elles, ce qui engendrerait des problèmes de multicolinéarité. Par exemple, dans la spécification a, on s'intéresse seulement au rôle de la santé subjective de la mère.

Pour les spécifications dans lesquelles nous régressons la santé de l'enfant sur la santé de sa mère, l'échantillon contient seulement des enfants qui vivaient avec leur mère en $t$-3 (ou $t$-4 ou $t$-5), c'est-à-dire dans une famille avec leur mère et leur père ou dans une famille monoparentale avec leur mère. Pour les spécifications dans lesquels nous régressons la santé de l'enfant sur la santé de son père, l'échantillon contient seulement des enfants qui vivaient leur père en $t$-3 (ou $t$ - 4 ou $t-5$ ), c'est-à-dire dans une famille avec leur mère et leur père ou dans une famille monoparentale avec leur père.

Pour les variables de santé subjective, digestive, et pondérale de l'enfant, pour lesquelles nous avons observé un gradient dans le tableau 2, spécification A, nous estimons deux modèles : 
d'abord nous ré-estimons le modèle (I) qui ne contrôle pas pour la santé des parents, mais cette fois en utilisant seulement les observations pour lesquelles les variables de santé des parents ne sont pas manquantes ; ensuite nous estimons le modèle (III) qui contrôle pour la santé des parents. Notez que dans l'encadré 3 nous commentons les restrictions d'échantillon. Dans le tableau 4, les colonnes (1), (5), et (7) contiennent les résultats de l'estimation du modèle (I), tandis que les colonnes (2), (6), et (8) présentent ceux du modèle (III). Les « contrôles 1 » sont inclus. Par construction, les coefficients du tableau 4, colonnes (1), (5), et (7), sont relativement proches de ceux du tableau 2, spécification A, les différences étant dues au fait que dans le tableau 4 l'échantillon est plus petit. La comparaison des gradients entre les colonnes (1) et (2), (5) et (6), et (7) et (8) permet de savoir si le gradient est robuste à la prise en compte de la santé des parents.

Pour les variables de faible morbidité et de santé respiratoire pour lesquelles nous ne trouvons pas de gradient dans le tableau 2, spécification A, nous estimons seulement le modèle qui contrôle pour la santé des parents. Les résultats sont présentés dans le tableau 4, colonnes (3) et (4).

On s'intéresse en premier lieu au gradient de santé subjective dans l'enfance. Dans la spécification a, colonnes (1) et (2), la variable expliquée est la santé subjective de l'enfant. Les résultats soulignent que lorsque l'on tient compte de la santé subjective de la mère, le gradient de santé subjective de l'enfant est divisé par plus de deux et perd sa significativité. De plus, dans la spécification b, colonne (2), le gradient de santé subjective est fortement réduit lorsque l'on inclut la santé subjective du père. Les spécifications c à j soulignent que l'inclusion des autres variables de santé des parents (faible morbidité, santé respiratoire, digestive, et pondérale) affecte moins, voire n'affecte pas, le gradient de santé subjective dans l'enfance.

Quelle que soit la variable de santé des parents incluse dans le modèle, les gradients de santé digestive et pondérale restent significatifs à $1 \%$. Par conséquent, l'effet du revenu familial sur la santé digestive et pondérale des enfants est indépendant de la santé des parents.

Le tableau 4 met aussi en lumière les liens intergénérationnels de santé. La santé subjective de la mère est positivement associée à la santé subjective de l'enfant. Cette corrélation se renforce avec le niveau de santé subjective de la mère, puisque les coefficients des variables de santé subjective de la mère augmentent lorsque le score de santé subjective de la mère croît. L'effet de la santé subjective de la mère semble assez important : en termes d'effet marginal, lorsque le score de santé subjective de la mère passe de 4 à 5 , la probabilité que le score de santé subjective de l'enfant soit égal à 5 augmente de 12,4 points de pourcentage, passant de $65,2 \%$ à 77,6\%. La santé subjective de la mère est également associée aux autres variables de santé de l'enfant, et cette association se renforce aussi avec l'état de santé de la mère. La santé subjective du père est aussi corrélée à la santé subjective, à la faible morbidité, et à la santé digestive de l'enfant. En termes d'effet marginal, lorsque le score de santé subjective du père passe de 4 à 5 , la probabilité que le score de santé subjective de l'enfant soit égal à 5 augmente de 12,9 points de pourcentage, passant de $59,8 \%$ à $72,7 \%$. 
Contrairement à la santé subjective de la mère, la santé subjective du père n'a pas d'effet significatif sur la santé pondérale de l'enfant.

De plus, la faible morbidité du père et de la mère est associée à toutes les variables de santé de l'enfant. La transmission de faible morbidité entre parents et enfant se renforce avec le score de faible morbidité des parents. La santé respiratoire (resp. digestive) des parents est positivement et significativement associée à la santé respiratoire (resp. digestive) de l'enfant.

La corpulence passe également d'une génération à la suivante, via la mère : lorsque la corpulence de la mère devient normale, la probabilité que la corpulence de l'enfant soit aussi normale augmente de 7,6 points de pourcentage, passant de 66,0\% à 73,6\%. En revanche la corpulence du père n'a pas d'effet significatif sur la santé pondérale de l'enfant. 
Tableau 4. L'effet du revenu et de la santé des parents sur la santé des enfants

\begin{tabular}{|c|c|c|c|c|c|c|c|c|}
\hline & (1) & (2) & (3) & (4) & $(5)$ & (6) & (7) & $(8)$ \\
\hline Variable expliquée & $\begin{array}{c}\text { Santé } \\
\text { subjective } \\
\text { Enfant } \\
\text { en } t\end{array}$ & $\begin{array}{c}\text { Santé } \\
\text { subjective } \\
\text { Enfant } \\
\text { en } t\end{array}$ & $\begin{array}{c}\text { Faible } \\
\text { morbidité } \\
\text { Enfant } \\
\text { en } t\end{array}$ & $\begin{array}{c}\text { Santé } \\
\text { respiratoire } \\
\text { Enfant } \\
\text { en } t\end{array}$ & $\begin{array}{c}\text { Santé } \\
\text { digestive } \\
\text { Enfant } \\
\text { en } t\end{array}$ & $\begin{array}{c}\text { Santé } \\
\text { digestive } \\
\text { Enfant } \\
\text { en } t\end{array}$ & $\begin{array}{c}\text { Santé } \\
\text { pondérale } \\
\text { Enfant } \\
\text { en } t\end{array}$ & $\begin{array}{c}\text { Santé } \\
\text { pondérale } \\
\text { Enfant } \\
\text { en } t\end{array}$ \\
\hline Méthode & $\begin{array}{c}\text { Probit } \\
\text { ordonné }\end{array}$ & $\begin{array}{c}\text { Probit } \\
\text { Ordonné }\end{array}$ & $\begin{array}{c}\text { Probit } \\
\text { ordonné }\end{array}$ & Probit & Probit & Probit & Probit & Probit \\
\hline \multicolumn{9}{|c|}{ Spécification a. Santé subjective de la mère en $t-3, t-4$, ou $t-5$} \\
\hline $\log ($ revenu moyen $)$ & $\begin{array}{c}0,165 * * * \\
(0,0446)\end{array}$ & $\begin{array}{c}0,0707 \\
(0,0459)\end{array}$ & $\begin{array}{l}-0,0370 \\
(0,0409)\end{array}$ & $\begin{array}{l}-0,0628 \\
(0,0549)\end{array}$ & $\begin{array}{c}0,184 * * * \\
(0,0530)\end{array}$ & $\begin{array}{c}0,147 * * * \\
(0,0536)\end{array}$ & $\begin{array}{c}0,161 * * * \\
(0,0514)\end{array}$ & $\begin{array}{c}0,142 * * * \\
(0,0521)\end{array}$ \\
\hline Santé subjective 2 - Mère & & $\begin{array}{c}0,0398 \\
(0,0712)\end{array}$ & $\begin{array}{c}0,100 \\
(0,0688)\end{array}$ & $\begin{array}{c}0,117 \\
(0,0807)\end{array}$ & & $\begin{array}{c}0,104 \\
(0,0839)\end{array}$ & & $\begin{array}{c}0,0550 \\
(0,0821)\end{array}$ \\
\hline Santé subjective 3 - Mère & & $\begin{array}{c}0,321 * * * \\
(0,0623)\end{array}$ & $\begin{array}{c}0,299 * * * \\
(0,0601)\end{array}$ & $\begin{array}{c}0,250 * * * \\
(0,0719)\end{array}$ & & $\begin{array}{l}0,181 * * \\
(0,0707)\end{array}$ & & $\begin{array}{c}0,117 * \\
(0,0697)\end{array}$ \\
\hline Santé subjective 4 - Mère & & $\begin{array}{c}0,531 * * * \\
(0,0645)\end{array}$ & $\begin{array}{c}0,366^{* * *} \\
(0,0614)\end{array}$ & $\begin{array}{c}0,334 * * * \\
(0,0752)\end{array}$ & & $\begin{array}{c}0,216 \text { *** } \\
(0,0733)\end{array}$ & & $\begin{array}{c}0,128 * \\
(0,0717)\end{array}$ \\
\hline Santé subjective 5 - Mère & & $\begin{array}{c}0,897 * * * \\
(0,0671)\end{array}$ & $\begin{array}{c}0,488 * * * \\
(0,0612)\end{array}$ & $\begin{array}{c}0,364 * * * \\
(0,0744)\end{array}$ & & $\begin{array}{c}0,410 * * * \\
(0,0734)\end{array}$ & & $\begin{array}{l}0,177 * * \\
(0,0709)\end{array}$ \\
\hline Observations & 6448 & 6448 & 6622 & 6622 & 6622 & 6622 & 6006 & 6006 \\
\hline \multicolumn{9}{|c|}{ Spécification b. Santé subjective du père en $t-3, t-4$, ou $t-5$} \\
\hline Log(revenu moyen) & $\begin{array}{c}0,179 * * * \\
(0,0471)\end{array}$ & $\begin{array}{l}0,122 * * \\
(0,0484)\end{array}$ & $\begin{array}{c}-0,00396 \\
(0,0429)\end{array}$ & $\begin{array}{l}-0,0312 \\
(0,0574)\end{array}$ & $\begin{array}{c}0,209 * * * \\
(0,0556)\end{array}$ & $\begin{array}{c}0,183 \text { *** } \\
(0,0562)\end{array}$ & $\begin{array}{c}0,176^{* * *} * \\
(0,0544)\end{array}$ & $\begin{array}{c}0,168 * * * \\
(0,0550)\end{array}$ \\
\hline Santé subjective 2 - Père & & $\begin{array}{l}0,00805 \\
(0,0757)\end{array}$ & $\begin{array}{l}0,00555 \\
(0,0720)\end{array}$ & $\begin{array}{l}-0,179 * \\
(0,0963)\end{array}$ & & $\begin{array}{c}0,261 * * * \\
(0,0913)\end{array}$ & & $\begin{array}{c}0,0495 \\
(0,0940)\end{array}$ \\
\hline Santé subjective 3 - Père & & $\begin{array}{c}0,0628 \\
(0,0666)\end{array}$ & $\begin{array}{l}-0,00247 \\
(0,0649)\end{array}$ & $\begin{array}{l}-0,0470 \\
(0,0889)\end{array}$ & & $\begin{array}{c}0,0860 \\
(0,0787)\end{array}$ & & $\begin{array}{c}0,0463 \\
(0,0827)\end{array}$ \\
\hline Santé subjective 4 - Père & & $\begin{array}{c}0,241 \text { *** } \\
(0,0689)\end{array}$ & $\begin{array}{c}0,102 \\
(0,0655)\end{array}$ & $\begin{array}{r}-0,00873 \\
(0,0905)\end{array}$ & & $\begin{array}{l}0,202 * * \\
(0,0802)\end{array}$ & & $\begin{array}{c}0,0705 \\
(0,0846)\end{array}$ \\
\hline Santé subjective 5 - Père & & $\begin{array}{c}0,596^{* * *} \\
(0,0701)\end{array}$ & $\begin{array}{c}0,214 * * * \\
(0,0653)\end{array}$ & $\begin{array}{c}0,0909 \\
(0,0903)\end{array}$ & & $\begin{array}{c}0,286 \text { *** } \\
(0,0786)\end{array}$ & & $\begin{array}{c}0,0607 \\
(0,0830)\end{array}$ \\
\hline Observations & 6071 & 6071 & 6236 & 6236 & 6236 & 6236 & 5656 & 5656 \\
\hline \multicolumn{9}{|c|}{ Spécification c. Faible morbidité de la mère en $t-3, t-4$, ou $t-5$} \\
\hline Log(revenu moyen) & $\begin{array}{c}0,167 * * * \\
(0,0438)\end{array}$ & $\begin{array}{c}0,150 * * * \\
(0,0438)\end{array}$ & $\begin{array}{c}0,000418 \\
(0,0394)\end{array}$ & $\begin{array}{l}-0,0429 \\
(0,0525)\end{array}$ & $\begin{array}{c}0,171 * * * \\
(0,0518)\end{array}$ & $\begin{array}{c}0,156^{* * * *} \\
(0,0519)\end{array}$ & $\begin{array}{c}0,170 * * * \\
(0,0503)\end{array}$ & $\begin{array}{c}0,167 * * * \\
(0,0503)\end{array}$ \\
\hline Faible morbidité 2 - Mère & & $\begin{array}{c}0,222 * * * \\
(0,0433)\end{array}$ & $\begin{array}{c}0,222 * * * \\
(0,0398)\end{array}$ & $\begin{array}{c}0,213 \text { *** } \\
(0,0514)\end{array}$ & & $\begin{array}{c}0,211 * * * \\
(0,0528)\end{array}$ & & $\begin{array}{c}0,0523 \\
(0,0503)\end{array}$ \\
\hline Faible morbidité 3 - Mère & & $\begin{array}{c}0,274 * * * \\
(0,0414)\end{array}$ & $\begin{array}{c}0,338 * * * \\
(0,0378)\end{array}$ & $\begin{array}{c}0,295 * * * \\
(0,0500)\end{array}$ & & $\begin{array}{c}0,256^{* * * *} \\
(0,0499)\end{array}$ & & $\begin{array}{c}0,0781 \\
(0,0482)\end{array}$ \\
\hline Faible morbidité 4 - Mère & & $\begin{array}{c}0,373 * * * \\
(0,0449)\end{array}$ & $\begin{array}{c}0,464 * * * \\
(0,0417)\end{array}$ & $\begin{array}{c}0,380 * * * \\
(0,0544)\end{array}$ & & $\begin{array}{c}0,302 * * * \\
(0,0564)\end{array}$ & & $\begin{array}{c}0,0860 * \\
(0,0519)\end{array}$ \\
\hline Faible morbidité 5 - Mère & & $\begin{array}{c}0,271 * * * \\
(0,0674)\end{array}$ & $\begin{array}{c}0,580 * * * \\
(0,0645)\end{array}$ & $\begin{array}{c}0,486^{* * * *} \\
(0,0915)\end{array}$ & & $\begin{array}{c}0,382 * * * \\
(0,0973)\end{array}$ & & $\begin{array}{c}0,0625 \\
(0,0831)\end{array}$ \\
\hline Observations & 6547 & 6547 & 6740 & 6740 & 6740 & 6740 & 6098 & 6098 \\
\hline \multicolumn{9}{|c|}{ Spécification d. Faible morbidité du père en $t-3, t-4$, ou $t-5$} \\
\hline $\log ($ revenu moyen $)$ & $\begin{array}{c}0,174 * * * \\
(0,0459)\end{array}$ & $\begin{array}{c}0,176^{* * *} \\
(0,0459)\end{array}$ & $\begin{array}{c}0,0277 \\
(0,0415)\end{array}$ & $\begin{array}{l}-0,0121 \\
(0,0549)\end{array}$ & $\begin{array}{c}0,192 * * * \\
(0,0544)\end{array}$ & $\begin{array}{c}0,186^{* * * *} \\
(0,0544)\end{array}$ & $\begin{array}{c}0,184 * * * \\
(0,0528)\end{array}$ & $\begin{array}{c}0,189 * * * \\
(0,0529)\end{array}$ \\
\hline Faible morbidité 2 - Père & & $\begin{array}{c}0,131 \text { *** } \\
(0,0505)\end{array}$ & $\begin{array}{c}0,239 * * * \\
(0,0471)\end{array}$ & $\begin{array}{c}0,0685 \\
(0,0605)\end{array}$ & & $\begin{array}{l}0,131 * * \\
(0,0634)\end{array}$ & & $\begin{array}{c}0,00786 \\
(0,0608)\end{array}$ \\
\hline Faible morbidité 3 - Père & & $\begin{array}{c}0,186^{* * *} \\
(0,0479)\end{array}$ & $\begin{array}{c}0,261 * * * \\
(0,0443)\end{array}$ & $\begin{array}{c}0,171 * * * \\
(0,0566)\end{array}$ & & $\begin{array}{l}0,145^{* *} \\
(0,0592)\end{array}$ & & $\begin{array}{c}0,0283 \\
(0,0567)\end{array}$ \\
\hline Faible morbidité 4 - Père & & $\begin{array}{c}0,269 * * * \\
(0,0499)\end{array}$ & $\begin{array}{c}0,376^{* * *} \\
(0,0463)\end{array}$ & $\begin{array}{c}0,305^{* * *} \\
(0,0592)\end{array}$ & & $\begin{array}{c}0,0162 \\
(0,0597)\end{array}$ & & $\begin{array}{c}0,113^{*} \\
(0,0591)\end{array}$ \\
\hline Faible morbidité 5 - Père & & $\begin{array}{c}0,247 * * * \\
(0,0724)\end{array}$ & $\begin{array}{c}0,545^{* * * *} \\
(0,0670)\end{array}$ & $\begin{array}{c}0,405^{* * *} \\
(0,0900)\end{array}$ & & $\begin{array}{c}0,311 \text { *** } \\
(0,0971)\end{array}$ & & $\begin{array}{c}0,0348 \\
(0,0865)\end{array}$ \\
\hline Observations & 6232 & 6232 & 6416 & 6416 & 6416 & 6416 & 5806 & 5806 \\
\hline
\end{tabular}




\begin{tabular}{|c|c|c|c|c|c|c|c|c|}
\hline Variable expliquée & $\begin{array}{c}\text { Santé } \\
\text { subjective } \\
\text { Enfant } \\
\text { en } t\end{array}$ & $\begin{array}{c}\text { Santé } \\
\text { subjective } \\
\text { Enfant } \\
\text { en } t\end{array}$ & $\begin{array}{l}\text { Faible } \\
\text { morbidité } \\
\text { Enfant } \\
\text { en } t\end{array}$ & $\begin{array}{c}\text { Santé } \\
\text { respiratoire } \\
\text { Enfant } \\
\text { en } t\end{array}$ & $\begin{array}{c}\text { Santé } \\
\text { digestive } \\
\text { Enfant } \\
\text { en } t\end{array}$ & $\begin{array}{c}\text { Santé } \\
\text { digestive } \\
\text { Enfant } \\
\text { en } t\end{array}$ & $\begin{array}{c}\text { Santé } \\
\text { pondérale } \\
\text { Enfant } \\
\text { en } t\end{array}$ & $\begin{array}{c}\text { Santé } \\
\text { pondérale } \\
\text { Enfant } \\
\text { en } t\end{array}$ \\
\hline Méthode & $\begin{array}{c}\text { Probit } \\
\text { ordonné }\end{array}$ & $\begin{array}{c}\text { Probit } \\
\text { Ordonné }\end{array}$ & $\begin{array}{c}\text { Probit } \\
\text { ordonné }\end{array}$ & Probit & Probit & Probit & Probit & Probit \\
\hline \multicolumn{9}{|c|}{ Spécification e. Santé respiratoire de la mère en $t-3, t-4$, ou $t-5$} \\
\hline Log(revenu moyen) & $\begin{array}{c}0,167 * * * \\
(0,0438)\end{array}$ & $\begin{array}{c}0,168 * * * \\
(0,0438)\end{array}$ & $\begin{array}{c}0,0204 \\
(0,0395)\end{array}$ & $\begin{array}{l}-0,0250 \\
(0,0525)\end{array}$ & $\begin{array}{c}0,171 \text { *** } \\
(0,0518)\end{array}$ & $\begin{array}{c}0,172 * * * \\
(0,0517)\end{array}$ & $\begin{array}{c}0,170 * * * \\
(0,0503)\end{array}$ & $\begin{array}{c}0,169 * * * \\
(0,0504)\end{array}$ \\
\hline Santé respiratoire - Mère & & $\begin{array}{c}0,223 * * * \\
(0,0382)\end{array}$ & $\begin{array}{c}0,218 * * * \\
(0,0362)\end{array}$ & $\begin{array}{c}0,310 * * * \\
(0,0453)\end{array}$ & & $\begin{array}{l}0,112 * * \\
(0,0487)\end{array}$ & & $\begin{array}{c}-0,0787 * \\
(0,0458)\end{array}$ \\
\hline Observations & 6547 & 6547 & 6740 & 6740 & 6740 & 6740 & 6098 & 6098 \\
\hline \multicolumn{9}{|c|}{ Spécification f. Santé respiratoire du père en $t-3, t-4$, ou $t-5$} \\
\hline Log(revenu moyen) & $\begin{array}{c}0,174 * * * \\
(0,0459)\end{array}$ & $\begin{array}{c}0,170 * * * \\
(0,0459)\end{array}$ & $\begin{array}{c}0,0149 \\
(0,0414)\end{array}$ & $\begin{array}{l}-0,0284 \\
(0,0548)\end{array}$ & $\begin{array}{c}0,192 * * * \\
(0,0544)\end{array}$ & $\begin{array}{c}0,193 * * * \\
(0,0545)\end{array}$ & $\begin{array}{c}0,184 * * * \\
(0,0528)\end{array}$ & $\begin{array}{c}0,187 * * * \\
(0,0529)\end{array}$ \\
\hline Santé respiratoire - Père & & $\begin{array}{c}0,187 * * * \\
(0,0440)\end{array}$ & $\begin{array}{c}0,190 * * * \\
(0,0407)\end{array}$ & $\begin{array}{c}0,270 * * * \\
(0,0526)\end{array}$ & & $\begin{array}{l}-0,0338 \\
(0,0578)\end{array}$ & & $\begin{array}{c}-0,0992 * \\
(0,0526)\end{array}$ \\
\hline Observations & 6232 & 6232 & 6416 & 6416 & 6416 & 6416 & 5806 & 5806 \\
\hline \multicolumn{9}{|c|}{ Spécification g. Santé digestive de la mère en $t-3, t-4$, ou $t-5$} \\
\hline Log(revenu moyen) & $\begin{array}{c}0,167 * * * \\
(0,0438)\end{array}$ & $\begin{array}{c}0,160 * * * \\
(0,0439)\end{array}$ & $\begin{array}{l}0,00764 \\
(0,0394)\end{array}$ & $\begin{array}{l}-0,0230 \\
(0,0523)\end{array}$ & $\begin{array}{c}0,171 \text { *** } \\
(0,0518)\end{array}$ & $\begin{array}{c}0,161 * * * \\
(0,0519)\end{array}$ & $\begin{array}{c}0,170 * * * \\
(0,0503)\end{array}$ & $\begin{array}{c}0,165 * * * \\
(0,0503)\end{array}$ \\
\hline Santé digestive - Mère & & $\begin{array}{c}0,124 * * * \\
(0,0390)\end{array}$ & $\begin{array}{c}0,192 * * * \\
(0,0366)\end{array}$ & $\begin{array}{l}-0,00278 \\
(0,0475)\end{array}$ & & $\begin{array}{c}0,218 * * * \\
(0,0544)\end{array}$ & & $\begin{array}{c}0,0826^{*} \\
(0,0457)\end{array}$ \\
\hline Observations & 6547 & 6547 & 6740 & 6740 & 6740 & 6740 & 6098 & 6098 \\
\hline \multicolumn{9}{|c|}{ Spécification $\mathrm{h}$. Santé digestive du père en $t-3, t-4$, ou $t-5$} \\
\hline Log(revenu moyen) & $\begin{array}{c}0,174 * * * \\
(0,0459)\end{array}$ & $\begin{array}{c}0,174 * * * \\
(0,0459)\end{array}$ & $\begin{array}{c}0,0204 \\
(0,0416)\end{array}$ & $\begin{array}{c}-0,0208 \\
(0,0549)\end{array}$ & $\begin{array}{c}0,192 * * * \\
(0,0544)\end{array}$ & $\begin{array}{c}0,193 * * * \\
(0,0543)\end{array}$ & $\begin{array}{c}0,184 * * * \\
(0,0528)\end{array}$ & $\begin{array}{c}0,184 * * * \\
(0,0528)\end{array}$ \\
\hline Santé digestive - Père & & $\begin{array}{c}0,0351 \\
(0,0394)\end{array}$ & $\begin{array}{c}0,120 * * * \\
(0,0359)\end{array}$ & $\begin{array}{l}0,0827 * \\
(0,0481)\end{array}$ & & $\begin{array}{l}0,135 * * \\
(0,0528)\end{array}$ & & $\begin{array}{l}-0,0217 \\
(0,0467)\end{array}$ \\
\hline Observations & 6232 & 6232 & 6416 & 6416 & 6416 & 6416 & 5806 & 5806 \\
\hline \multicolumn{9}{|c|}{ Spécification i. Santé pondérale de la mère en $t-3, t-4$, ou $t-5$} \\
\hline Log(revenu moyen) & $\begin{array}{c}0,161 * * * \\
(0,0442)\end{array}$ & $\begin{array}{c}0,155^{* * *} \\
(0,0445)\end{array}$ & $\begin{array}{l}0,00801 \\
(0,0402)\end{array}$ & $\begin{array}{c}-0,0273 \\
(0,0534)\end{array}$ & $\begin{array}{c}0,181 * * * \\
(0,0523)\end{array}$ & $\begin{array}{c}0,164 * * * \\
(0,0527)\end{array}$ & $\begin{array}{c}0,171 * * * \\
(0,0508)\end{array}$ & $\begin{array}{c}0,141 * * * \\
(0,0510)\end{array}$ \\
\hline Santé pondérale - Mère & & $\begin{array}{c}0,0375 \\
(0,0339)\end{array}$ & $\begin{array}{c}0,0713 * * \\
(0,0309)\end{array}$ & $\begin{array}{c}0,0265 \\
(0,0417)\end{array}$ & & $\begin{array}{c}0,113 \text { *** } \\
(0,0405)\end{array}$ & & $\begin{array}{c}0,219 * * * \\
(0,0394)\end{array}$ \\
\hline Observations & 6451 & 6451 & 6637 & 6637 & 6637 & 6637 & 6015 & 6015 \\
\hline \multicolumn{9}{|c|}{ Spécification j. Santé pondérale du père en $t-3, t-4$, ou $t-5$} \\
\hline Log(revenu moyen) & $\begin{array}{c}0,173 \text { *** } \\
(0,0461)\end{array}$ & $\begin{array}{c}0,178 * * * \\
(0,0462)\end{array}$ & $\begin{array}{c}0,0171 \\
(0,0417)\end{array}$ & $\begin{array}{l}-0,0150 \\
(0,0553)\end{array}$ & $\begin{array}{c}0,197 * * * \\
(0,0542)\end{array}$ & $\begin{array}{c}0,192 * * * \\
(0,0544)\end{array}$ & $\begin{array}{c}0,183 * * * \\
(0,0531)\end{array}$ & $\begin{array}{c}0,182 * * * \\
(0,0531)\end{array}$ \\
\hline Santé pondérale - Père & & $\begin{array}{c}-0,0624^{*} \\
(0,0322)\end{array}$ & $\begin{array}{c}0,0679 * * \\
(0,0293)\end{array}$ & $\begin{array}{l}-0,00468 \\
(0,0387)\end{array}$ & & $\begin{array}{l}0,0759 * \\
(0,0391)\end{array}$ & & $\begin{array}{c}0,0160 \\
(0,0376)\end{array}$ \\
\hline Observations & 6160 & 6160 & 6335 & 6335 & 6335 & 6335 & 5743 & 5743 \\
\hline
\end{tabular}

Lecture : pour les spécifications a, c, e, g, et i, les échantillons comprennent des enfants qui vivaient dans le même ménage que leur mère en $t-3, t-4$, ou $t-5$. Pour les spécifications $\mathrm{b}, \mathrm{d}, \mathrm{f}, \mathrm{h}$, et $\mathrm{j}$, les échantillons comprennent des enfants qui vivaient dans le même ménage que leur père en $t-3, t-4$, ou $t-5$. La santé de l'enfant, le revenu, et les variables de contrôle sont mesurés à la date $t$, tandis que la santé des parents est mesurée à $t-3, t$ 4 , ou $t-5$. Les « contrôles 1 » sont inclus, c'est-à-dire : le type de ménage, le sexe de l'enfant, des variables indicatrices pour l'âge de l'enfant, l'âge de la mère et du père interagis avec leur présence, le logarithme de la taille du ménage, l'identité du répondant aux questions de santé de l'enfant, et l'année de l'enquête. Les écartstypes sont donnés entre parenthèses.

Seuils de significativité du test de rejet de l'hypothèse de nullité du coefficient : *** $1 \%, * * 5 \%, * 10 \%$.

Champ : ménages ordinaires vivant en France métropolitaine.

Source : vagues 1994-2008 de l'enquête Santé et Protection Sociale (ESPS) de l'IRDES, calcul des auteurs. 
Pour mieux connaître le rôle de la santé subjective des parents dans celle des enfants, nous réestimons le modèle de santé subjective en contrôlant à la fois pour la santé subjective de la mère et du père, dans le tableau 5. Les estimations incluent aussi les « contrôles 1 ».

L'échantillon contient des enfants qui vivaient dans une famille avec leur mère et leur père 3 à 5 ans avant l'enquête. Dans la colonne (1), nous mesurons le gradient de santé pour ce nouvel échantillon. Dans la colonne (2), nous ajoutons les variables de santé subjective des parents comme variables explicatives. On observe alors que le gradient de santé subjective de l'enfant est divisé par plus de trois et perd sa significativité. De plus, la très bonne santé subjective du père influence la santé de l'enfant indépendamment de la santé subjective de la mère.

Le tableau 5 contient en outre les coefficients des variables de contrôle. La plupart de ces contrôles ne sont pas significatifs. Cela est lié à la faible puissance statistique du modèle, qui est estimé sur un échantillon relativement petit. En outre, les coefficients sur le statut du ménage (famille monoparentale avec la mère ou avec le père) ne sont pas significatifs, parce que dans notre échantillon très peu de familles comprenant les deux parents sont devenues des familles monoparentales entre $t$ - 3 (ou $t$ - 4 ou $t-5$ ) et $t$. Les filles sont en moins bonne santé subjective que les garçons. On observe une dégradation continue de la santé subjective pour les adolescents de plus de 13 ans. Les enfants qui déclarent eux-mêmes leur état de santé se disent en moins bonne santé subjective. Ainsi, les résultats sur les variables de contrôle sont cohérents avec ceux présentés dans le tableau 2. 
Tableau 5. Le gradient et la santé subjective des parents

\begin{tabular}{|c|c|c|}
\hline Variable expliquée & $\begin{array}{c}\text { (1) } \\
\text { Santé subjective - Enfant } \\
\text { en } t\end{array}$ & $\begin{array}{c}(2) \\
\text { Santé subjective - Enfant } \\
\text { en } t\end{array}$ \\
\hline Méthode & Probit ordonné & Probit ordonné \\
\hline $\log ($ revenu moyen $)$ & $\begin{array}{c}0,1827 * * * \\
(0,0475)\end{array}$ & $\begin{array}{c}0,0558 \\
(0,0501)\end{array}$ \\
\hline \multicolumn{3}{|c|}{ Santé subjective des parents en $t-3, t-4$, ou $t-5$} \\
\hline Santé subjective 2 - Mère & & $\begin{array}{c}0,0538 \\
(0,0758)\end{array}$ \\
\hline Santé subjective 3 - Mère & & $\begin{array}{c}0,3095 * * * \\
(0,0665)\end{array}$ \\
\hline Santé subjective 4 - Mère & & $\begin{array}{c}0,4956 * * * \\
(0,0691)\end{array}$ \\
\hline Santé subjective 5 - Mère & & $\begin{array}{c}0,7579 * * * \\
(0,0736)\end{array}$ \\
\hline Santé subjective 2 - Père & & $\begin{array}{l}-0,0230 \\
(0,0779)\end{array}$ \\
\hline Santé subjective 3 - Père & & $\begin{array}{l}-0,0042 \\
(0,0685)\end{array}$ \\
\hline Santé subjective 4 - Père & & $\begin{array}{c}0,1166 \\
(0,0715)\end{array}$ \\
\hline Santé subjective 5 - Père & & $\begin{array}{c}0,3371 * * * \\
(0,0744)\end{array}$ \\
\hline Variables de contrôle en $t$ & & \\
\hline Famille monoparentale avec la mère & $\begin{array}{c}0,1736 \\
(0,2150)\end{array}$ & $\begin{array}{c}0,2850 \\
(0,2120)\end{array}$ \\
\hline Famille monoparentale avec le père & $\begin{array}{c}0,3689 \\
(0,3358)\end{array}$ & $\begin{array}{c}0,4270 \\
(0,3376)\end{array}$ \\
\hline Fille & $\begin{array}{c}-0,0737 * * \\
(0,0328)\end{array}$ & $\begin{array}{c}-0,0835 * * \\
(0,0325)\end{array}$ \\
\hline Moins d'1 an & $\begin{array}{l}-0,0408 \\
(0,3839)\end{array}$ & $\begin{array}{c}0,0483 \\
(0,4391)\end{array}$ \\
\hline 1 an & $\begin{array}{c}0,0377 \\
(0,4079)\end{array}$ & $\begin{array}{c}0,1199 \\
(0,4620)\end{array}$ \\
\hline 2 ans & $\begin{array}{l}-0,0289 \\
(0,2157)\end{array}$ & $\begin{array}{c}0,0461 \\
(0,1985)\end{array}$ \\
\hline 3 ans & $\begin{array}{c}-0,4806 * * * \\
(0,1655)\end{array}$ & $\begin{array}{c}-0,5310 * * * \\
(0,1688)\end{array}$ \\
\hline 4 ans & $\begin{array}{l}-0,1362 \\
(0,0939)\end{array}$ & $\begin{array}{l}-0,1242 \\
(0,0964)\end{array}$ \\
\hline 5 ans & $\begin{array}{l}-0,0590 \\
(0,0789)\end{array}$ & $\begin{array}{l}-0,0896 \\
(0,0822)\end{array}$ \\
\hline 6 ans & $\begin{array}{l}-0,0006 \\
(0,0842)\end{array}$ & $\begin{array}{l}-0,0011 \\
(0,0868)\end{array}$ \\
\hline 7 ans & $\begin{array}{l}-0,0892 \\
(0,0821)\end{array}$ & $\begin{array}{l}-0,1034 \\
(0,0843)\end{array}$ \\
\hline
\end{tabular}




\begin{tabular}{|c|c|c|}
\hline 8 ans & $\begin{array}{c}0,0607 \\
(0,0828)\end{array}$ & $\begin{array}{c}0,0525 \\
(0,0846)\end{array}$ \\
\hline \multirow[t]{2}{*}{9 ans } & $-0,0733$ & $-0,0526$ \\
\hline & $(0,0811)$ & $(0,0829)$ \\
\hline \multirow[t]{2}{*}{11 ans } & $-0,1985^{* *}$ & $-0,2175^{* * *}$ \\
\hline & $(0,0828)$ & $(0,0837)$ \\
\hline \multirow{2}{*}{12 ans } & $-0,0675$ & $-0,0693$ \\
\hline & $(0,0797)$ & $(0,0817)$ \\
\hline \multirow[t]{2}{*}{13 ans } & $-0,1483^{*}$ & $-0,1373 *$ \\
\hline & $(0,0769)$ & $(0,0796)$ \\
\hline \multirow[t]{2}{*}{14 ans } & $-0,1861 * *$ & $-0,1968 * *$ \\
\hline & $(0,0817)$ & $(0,0840)$ \\
\hline \multirow[t]{2}{*}{15 ans } & $-0,2560 * * *$ & $-0,2504 * * *$ \\
\hline & $(0,0816)$ & $(0,0844)$ \\
\hline \multirow[t]{2}{*}{16 ans } & $-0,3642 * * *$ & $-0,3427 * * *$ \\
\hline & $(0,0807)$ & $(0,0826)$ \\
\hline \multirow[t]{2}{*}{17 ans } & $-0,3826 * * *$ & $-0,3598 * * *$ \\
\hline & $(0,0843)$ & $(0,0854)$ \\
\hline \multirow[t]{2}{*}{ Age - Mère } & 0,0035 & 0,0076 \\
\hline & $(0,0050)$ & $(0,0051)$ \\
\hline \multirow[t]{2}{*}{ Age - Père } & 0,0046 & $0,0085 * *$ \\
\hline & $(0,0043)$ & $(0,0042)$ \\
\hline \multirow[t]{2}{*}{ Log(taille du ménage) } & $0,2464 * * *$ & 0,1426 \\
\hline & $(0,0898)$ & $(0,0903)$ \\
\hline \multirow[t]{2}{*}{ Enfant déclare sa santé } & $-0,2311 * * *$ & $-0,2381 * * *$ \\
\hline & $(0,0450)$ & $(0,0456)$ \\
\hline Inconnu déclare la santé & $-0,1587$ & $-0,1894 *$ \\
\hline de l'enfant & $(0,1037)$ & $(0,1021)$ \\
\hline Observations & 5938 & 5938 \\
\hline
\end{tabular}

Lecture : L'échantillon est constitué des enfants qui vivaient avec leurs deux parents en $t-3, t-4$, ou $t-5$. La santé de l'enfant, le revenu, et les variables de contrôle sont mesurés à la date $t$, tandis que la santé des parents est mesurée à $t-3, t-4$, ou $t-5$. Les « contrôles 1 », qui sont mesurés à la date $t$, sont inclus, c'est-à-dire : le type de ménage, le sexe de l'enfant, des variables indicatrices pour l'âge de l'enfant, l'âge de la mère et du père interagis avec leur présence, le logarithme de la taille du ménage, l'identité du répondant aux questions de santé de l'enfant, et l'année de l'enquête. Les catégories de référence sont : les familles avec la mère et le père, les enfants de 10 ans, les parents déclarant la santé de l'enfant. Les écarts-types sont donnés entre parenthèses.

Seuils de significativité du test de rejet de l'hypothèse de nullité du coefficient: $* * * 1 \%, * * 5 \%, * 10 \%$. Champ : ménages ordinaires vivant en France métropolitaine.

Source : vagues 1994-2008 de l'enquête Santé et Protection Sociale (ESPS) de l'IRDES, calculs des auteurs.

\section{Discussion et conclusion}

Notre article examine l'association entre le revenu familial, la santé des enfants, et la santé des parents, en France, à partir des données ESPS de 1994 à 2008. Nous trouvons d'abord une association positive entre le revenu familial moyen et la santé subjective de l'enfant. Ce 
résultat fait écho aux résultat d'une étude récente sur la France (Apouey et Geoffard, 2014) et plus généralement, de travaux portant sur d'autres pays développés, qui mettent en évidence un gradient de santé subjective dans l'enfance, souvent en dépit de systèmes de soins universels (Case et al., 2002 ; Currie et Stabile, 2003 ; Reinhold et Jürges, 2012).

En outre, notre analyse souligne l'absence de corrélation entre le revenu familial et la morbidité de l'enfant, lorsque celle-ci est mesurée par le nombre de maladies. Ce résultat pourrait s'expliquer par la plus faible fréquence des visites chez le médecin des enfants de milieux défavorisés, qui entraînerait mécaniquement une moins bonne information sur les problèmes de santé objectifs, et donc une sous-déclaration de ces problèmes. Par conséquent, le gradient de morbidité pourrait être sous-estimé. Cependant, on trouve une association positive et significative entre revenu familial et santé digestive. De plus, le revenu moyen joue un rôle protecteur contre le risque de sous-poids, surpoids, et obésité des enfants.

Nous nous intéressons ensuite au rôle joué par la santé des parents dans le gradient de santé et à la transmission intergénérationnelle de la santé dans l'enfance. A notre connaissance, ces sujets ont fait l'objet de très peu de travaux de recherche, et en particulier n'ont pas été étudiés à partir de données françaises. La santé des parents pourrait être un mécanisme sousjacent au gradient dans deux cas : d'une part, si le revenu a un effet sur la santé des parents qui elle-même influence celle des enfants, et d'autre part, si la santé des parents est un facteur omis dans le modèle du gradient (Case et al., 2002). Bien que nous ne soyons pas en mesure de distinguer ces deux mécanismes, nous pouvons cependant examiner le rôle qu'ils jouent pris simultanément. Lorsque l'on contrôle pour la santé subjective de la mère, le gradient de santé subjective dans l'enfance baisse de moitié et perd sa significativité. Lorsque l'on contrôle pour la santé subjective du père, le gradient de santé subjective dans l'enfance baisse fortement (et sa significativité passe de $1 \%$ à 5\%). Ces résultats peuvent être interprétés de plusieurs manières : d'abord ils pourraient refléter les biais de déclaration dans la variable de santé subjective des enfants, puisque ce sont les parents qui répondent à la fois aux questions sur leur propre santé et aux questions sur la santé des enfants ; ensuite, il est possible que le gradient de santé subjective de l'enfant capture en partie une corrélation fallacieuse due à l'omission des variables de santé subjective des parents ; finalement, le gradient pourrait représenter un effet causal du revenu sur la santé subjective de l'enfant, mais cet effet transiterait en grande partie par la santé subjective des parents.

Les gradients de santé digestive et pondérale dans l'enfance sont robustes à l'inclusion de contrôles pour la santé des parents, ce qui suggère que le revenu a un effet sur la santé digestive et pondérale des enfants qui est indépendant de celui de la santé des parents. Nos résultats confirment ceux de Propper et al. (2007) obtenus sur données britanniques, et les prolongent en montrant d'une part qu'ils sont valables pour des enfants de tous âges en France, et d'autre part qu'ils sont valides lorsque l'on considère la santé du père.

\footnotetext{
${ }^{8}$ Apouey et Geoffard (2014) utilisent une variable de santé subjective de l'enfant différente de celle employée ici. Il s'agit de la santé subjective recommandée par l'Organisation Mondiale de la Santé dans sa version européenne, qui se trouve dans les données ESPS à partir de 2004 seulement. La note de santé subjective utilisée ici est disponible pour les années antérieures et couvre donc un échantillon d'enfants plus grand.
} 
Notre travail se concentre sur le rôle de la santé des parents dans le gradient revenu / santé des enfants. Cependant, des mécanismes plus complexes pourraient expliquer ce gradient. Par exemple, le revenu des parents pourrait avoir un effet sur le renoncement aux soins des parents, qui influencerait à son tour l'état de santé des parents, qui lui-même aurait un impact sur la santé des enfants ${ }^{9}$. La variable de renoncement aux soins est disponible en 2006 et 2008, ce qui nous permet de ré-estimer le gradient en contrôlant pour le renoncement aux soins des parents, en plus des variables de santé des parents. Cette analyse suggère le rôle potentiellement important du renoncement aux soins des parents dans le gradient. Cependant, la question du renoncement aux soins souffre potentiellement de biais de déclaration importants. En outre, puisque les données de renoncement ne sont disponibles qu'en 2006 et 2008, cette analyse ne peut être réalisée que pour un échantillon d'enfants de petite taille, ce qui pose la question de la représentativité des résultats. Des données plus riches permettront un jour de reprendre cette analyse de façon plus rigoureuse.

Notre étude de l'association entre revenu familial, santé des parents, et santé des enfants est réalisée à partir des données en coupe de l'ESPS, entre 1994 à 2008. Nos résultats donnent le gradient moyen ainsi que le niveau moyen de transmission intergénérationnelle de la santé, sur cette période. Pour prolonger notre étude, un travail intéressant consisterait à examiner si l'association entre nos variables d'intérêt a évolué au cours de la période, dans un contexte de changement dans l'accès au système de soins. Une première analyse, dans laquelle nous interagissons la variable de revenu avec une variable temporelle, indique que les gradients de faible morbidité et de santé respiratoire, digestive, et pondérale sont restés stables au cours du temps, tandis que le gradient de santé subjective a diminué. Des analyses plus fines sont nécessaires sur ce point.

Nos analyses mettent en lumière une forte transmission intergénérationnelle de la santé subjective, de la morbidité, et de la corpulence, entre parents et enfants. Dans ce contexte, des politiques qui amélioreraient les différentes composantes de la santé des parents pourraient s'avérer plus efficaces que prévu, dans la mesure où elles pourraient bénéficier non pas seulement aux adultes directement visés, mais aussi à leurs enfants.

\footnotetext{
${ }^{9}$ Voir Chaupain-Guillot et al. (2014) pour un travail récent sur le renoncement aux soins en France.
} 


\section{Remerciements}

Les données des ESPS ont été fournies par l'IRDES. Les calculs, analyses, et interprétations présentés dans l'article n'engagent que les auteurs. Les auteurs remercient deux référés anonymes, Thomas Barnay, Damien Bricard, Emmanuelle Cambois, Carine Franc, Florence Jusot, Serge Paugam, Sandy Tubeuf, Yann Videau, et les participants à l'Atelier sur les Inégalités Sociales de Santé de 2013 (Paris School of Economics), et aux Journées des Economistes de la Santé Français de 2013, pour leurs commentaires pertinents. 


\section{BIBLIOGRAPHIE}

Ahlburg D. (1998), « Intergenerational transmission of health », American Economic Review, 88(2), pp. 265-270.

Apouey B. H. (2010), « On measuring and explaining socioeconomic polarization in health with an application to French data », Review of Income and Wealth, 56(1), pp. 141-170.

Apouey B. et Clark A. E. (sous presse), « Winning big but feeling no better? The effect of lottery prizes on physical and mental health », Health Economics.

Apouey B. et Geoffard P.-Y. (2013), « Family income and child health in the UK », Journal of Health Economics, 22(4), pp. 715-727.

Apouey B. et Geoffard P.-Y. (2014), « Child health and access to health care in France: evidence on the role of family income », Revue d'Epidémiologie et de Santé Publique, 62(3), pp. 170-190.

Apouey B. et Silber J. (2013), « Inequality and bi-polarization in socioeconomic status and health: ordinal approaches », Research on Economic Inequality, volume 21 « Health and Inequality », chapitre 4. Editeurs : Rosa-Dias P et O'Donnell O.

Blanpain N. (2011), « L'espérance de vie s'accroît, les inégalités sociales face à la mort demeurent », INSEE Première.

Bricard D. et Jusot F. (2012), « Milieu d'origine, situation sociale et parcours tabagique en France », Economie Publique, pp. 28-29.

Cambois E. et Jusot F. (2011), « Contribution of lifelong adverse experiences to social health inequalities: findings from a population survey in France », European Journal of Public Health, 21(5), pp. 667-673.

Case A., Lee D. et Paxson C. (2008), « The income gradient in children's health: a comment on Currie, Shields and Wheatley Price », Journal of Health Economics, 27(3), pp. 801-807.

Case A., Lubotsky D. et Paxson C. (2002), « Economic status and health in childhood: the origins of the gradient », American Economic Review, 92(5), pp. 1308-1334.

Chaupain-Guillot S., Guillot O. et Jankeliowitch-Laval E. (2014), « Le renoncement aux soins médicaux et dentaires : une analyse à partir des données de l'enquête SRCV », Economie et Statistique, n 469-470, pp. 169-197.

Cole T. J. et Lobstein T. (2012), « Extended international (IOTF) body mass index cut-offs for thinness, overweight and obesity », Pediatric Obesity, 7, pp. 284-294.

Coneus K. et Spiess C. K. (2012), « The intergenerational transmission of health in early childhood-Evidence from the German Socio-Economic Panel Study », Economics \& Human Biology, 10(1), pp. 89-97. 
Currie A., Shields M. A. et Price S. W. (2007), « The child health/family income gradient: evidence from England », Journal of Health Economics, 26(2), pp. 213-232.

Currie C., Zanotti C. et Morgan A. (2012), Social determinants of health and well-being among young people. Health Behaviour in School-aged Children (HBSC) study: international report from the 2009/2010 survey. Copenhague.

Currie J. et Moretti E. (2007), « Biology as destiny? Short and long-run determinants of intergenerational transmission of birth weight », Journal of Labor Economics, 25, pp. 231264.

Currie J. et Stabile M. (2003), « Socioeconomic status and child health: why is the relationship stronger for older children? », American Economic Review, 93(5), pp. 18131823.

Fletcher J., et Wolfe B. L. (sous presse), « Increasing our understanding of the healthincome gradient », Health Economics.

Frijters P., Haisken-DeNew J. P. et Shields M. A. (2005), « The causal effect of income on health: Evidence from German reunification », Journal of Health Economics, 24(5), pp. 9971017.

Gardner J. et Oswald A. (2007), « Money and mental wellbeing: a longitudinal study of medium-sized lottery wins », Journal of Health Economics, 26, pp. 49-60.

Grossman M. (2000), The human capital model. Handbook of Health Economics, 1, pp. 347408.

Kim B. et Ruhm C. J. (2012), « Inheritances, health and death », Health Economics, 21(2), pp. 127-144.

Klein-Platat C., Wagner A., Haan M. C., Arveiler D., Schlienger J. L. et Simon, C. (2003), « Prevalence and sociodemographic determinants of overweight in young French adolescents », Diabetes/Metabolism Research and Reviews, 19(2), pp. 153-158.

Kuehnle D. (2013), « The causal effect of family income on child health: a re-examination using an instrumental variables approach », Melbourne Institute Working Paper Series.

Lindahl M. (2005), « Estimating the effect of income on health and mortality using lottery prizes as an exogenous source of variation in income », Journal of Human Resources, 40(1), pp. 144-168.

Marmot M. et Bobak M. (2000), « International comparators and poverty and health in Europe », British Medical Journal, 321, pp. 1124-1128.

Propper C., Rigg J. et Burgess S. (2007), « Child health: evidence on the roles of family income and maternal mental health from a UK birth cohort », Health Economics, 16(11), pp. $1245-1269$. 
Reinhold S. et Jürges H. (2012), « Parental income and child health in Germany » Health Economics, 21(5), pp. 562-579.

Tubeuf S. (2009), « Les inégalités de santé selon le revenu en France en 2004 :

décomposition et explications », Revue d'Épidémiologie et de Santé Publique, 57(5), pp. 319328.

Tursz A. (2000), La santé de l'enfant. In D. Fassin, H. Grandjean, \& M. Kaminskidu (Eds.), Les inégalités sociales de santé (pp. 193-206). La Découverte, Recherches.

Van Doorslaer E. et Koolman X. (2004), « Explaining the differences in income-related health inequalities across European countries. Health Economics, 13(7), pp. 609-628. 


\section{Encadré 1. Les relations entre revenu familial et santé des enfants et des parents}

Les relations entre le revenu familial et la santé des enfants et des parents peuvent être schématisées de la façon suivante :

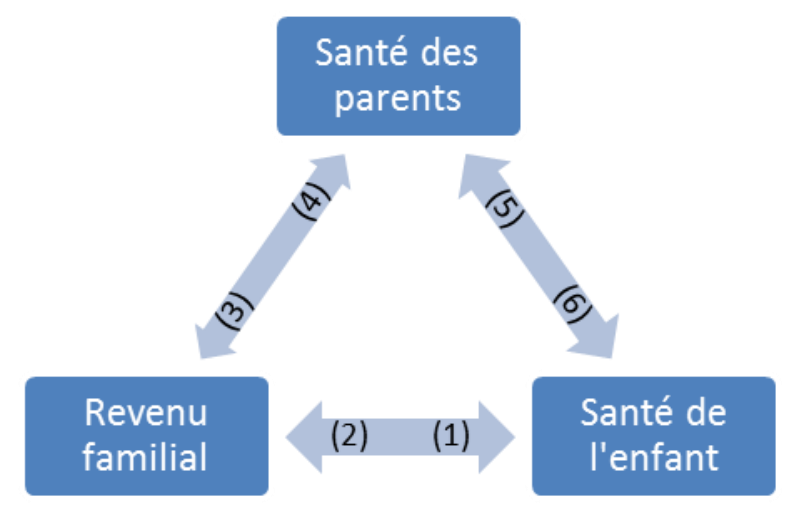

Flèche (1). L'effet du revenu familial sur la santé des enfants. C'est l'effet que les travaux récents sur le gradient de santé dans l'enfance souhaitent mesurer. Pour identifier cet impact, on peut d'une part tenter de tirer parti de la richesse de données d'observation, comme nous tentons de le faire dans notre article. On peut d'autre part instrumenter le revenu familial, par exemple par les conditions d'emploi sur le marché du travail local (Kuehnle, 2013).

Flèche (2). L'effet de la santé de l'enfant sur le revenu familial. Dans les pays développés dans lesquels les enfants ne travaillent pas, la santé de l'enfant peut théoriquement aussi influencer le revenu familial, mais de façon indirecte : les parents d'un enfant en mauvaise santé pourraient par exemple décider de ne pas travailler ou de travailler moins pour pouvoir s'occuper de leur enfant. Notons que certaines bases de données comme la Family and Children Study pour la Grande Bretagne contiennent des informations détaillées sur les raisons de la non-participation ou de la faible participation des adultes au marché du travail, ce qui permet de quantifier cet effet (Apouey et Geoffard, 2013). La littérature conclut que l'impact de la santé des enfants sur le revenu familial est faible et négligeable en population générale (Apouey et Geoffard, 2013 ; Case et al., 2002). Nous négligeons donc le canal (2) dans l'analyse présentée dans le présent article.

Flèche (3). L'effet de la santé des parents sur le revenu familial. Un tel effet apparaît lorsque les parents peuvent travailler davantage et percevoir un revenu plus élevé parce qu'ils sont en meilleure santé.

Flèche (4). L'effet du revenu familial sur la santé des parents. L'impact du revenu sur la santé des adultes a fait l'objet d'un certains nombres d'études, qui utilisent des chocs exogènes de revenu pour mesurer la causalité en question (Apouey et Clark, 2014 ; Kim et Ruhm, 2012). 
Flèche (5). L'effet de la santé des enfants sur la santé des parents. Dans notre article, nous essayons d'éliminer cet effet causal en utilisant des variables de santé des parents qui sont mesurées plusieurs années avant la santé des enfants.

Flèche (6). L'effet de la santé des parents sur la santé des enfants. C'est la transmission intergénérationnelle de la santé, qui opère d'au moins deux façons. D'une part, cette transmission peut refléter la transmission des gènes entre parents et enfants (biologiques). D'autre part, elle peut être due au fait que les parents qui sont en meilleure santé sont en mesure de mieux s'occuper de la santé de leurs enfants.

Lorsque l'on étudie le gradient revenu / santé des enfants, la santé des parents est un facteur par lequel transite l'effet causal du revenu sur la santé des enfants si les canaux (4) et (6) sont tous deux importants, tandis que la santé des parents joue un rôle de facteur omis si les canaux (3) et (6) opèrent simultanément. Quand l'on se penche sur la transmission intergénérationnelle de la santé, c'est la flèche (6) qui retient notre attention. 


\section{Encadré 2. L'indicateur de faible morbidité de l'enfant}

Cet encadré présente la construction de notre indicateur de faible morbidité de l'enfant. Les parents (ou l'enfant lui-même) déclarent si l'enfant (s'il) souffre de certaines maladies. Dans les données originelles, les maladies déclarées sont codées selon la Classification Internationale des Maladies, $9^{\text {ème }}$ révision (CIM-9) dans la vague 1998, et $10^{\text {ème }}$ révision (CIM-10) dans les vagues 2000-2008. Nous avons réalisé un travail d'uniformisation pour recoder les maladies selon la CIM-10 dans la vague 1998. Au final, nous savons si chaque enfant est atteint des maladies suivantes de la CIM-10 :

(I) Certaines maladies infectieuses et parasitaires ;

(II) Tumeurs ;

(III) Maladies du sang et des organes hématopoïétiques et certains troubles du système immunitaire ;

(IV) Maladies endocriniennes, nutritionnelles et métaboliques ;

(V) Troubles mentaux et du comportement ;

(VI) Maladies du système nerveux ;

(VII) Maladies de l'œil et de ses annexes ;

(VIII) Maladies de l'oreille et de l'apophyse mastoïde ;

(IX) Maladies de l'appareil circulatoire ;

(X) Maladies de l'appareil respiratoire ;

(XI) Maladies de l'appareil digestif ;

(XII) Maladies de la peau et du tissu cellulaire sous-cutané ;

(XIII) Maladies du système ostéo-articulaire, des muscles et du tissu conjonctif ;

(XIV) Maladies de l'appareil génito-urinaire ;

(XV) Grossesse, accouchement et puerpéralité ;

(XVI) Certaines affections dont l'origine se situe dans la période périnatale ;

(XVII) Malformations congénitales et anomalies chromosomiques ;

(XVIII) Symptômes, signes et résultats anormaux d'examens cliniques et de laboratoire, non classés ailleurs ;

(XIX) Lésions traumatiques, empoisonnements et certaines autres conséquences de causes externes.

L'indicateur de faible morbidité est une fonction du nombre de maladies déclarées.

Notons que nous avons éliminé de la liste les chapitres suivants de la CIM-10, parce qu'ils ne correspondent pas à une maladie précise :

(XX) Causes externes de morbidité et de mortalité ;

(XXI) Facteurs influant sur l'état de santé et motifs de recours aux services de santé ;

(XXII) Codes d'utilisation particulière. 


\section{Encadré 3. Les échantillons inclus et exclus dans le tableau 4}

Cet encadré commente sur les restrictions d'échantillon opérées dans le tableau 4, par rapport au tableau 2.

On prend l'exemple de la santé subjective de l'enfant et de la santé subjective de la mère. On appelle «échantillon total » l'échantillon utilisé dans le tableau 2, spécification A, colonne (1), qui contient tous les enfants observés à la date $t$. Il compte 11889 enfants.

On appelle «échantillon d'inclus » l'échantillon utilisé dans le tableau 4, spécification a, colonne (1) ou (2), qui contient les enfants qui remplissent toutes les conditions suivantes : en $t-3$ (ou $t$-4 ou $t-5$ ), leur ménage a été interrogé, ils étaient déjà nés, ils vivaient avec leur mère, et leur mère a répondu à la question sur sa propre santé subjective. L'échantillon d'inclus contient 6448 observations.

L'échantillon « d'exclus » est égal à l'échantillon total moins l'échantillon inclus, et compte 5441 enfants. Les enfants exclus le sont pour deux types de raisons :

- D'abord, des raisons liées à la constitution de l'échantillon : en effet ces enfants sont ceux dont le ménage n'a pas été interrogé en $t$-3 (ou $t-4$ ou $t-5$ ), qui n'étaient pas encore nés, ou qui ne vivaient pas avec leur mère en $t-3$ (ou $t-4$ ou $t-5$ ). Ces exclusions sont mécaniques et ne posent pas de problèmes particuliers pour notre étude.

- Ensuite, des raisons liées à la non-réponse des mères : en effet, les enfants dont la mère a été interrogée sur sa propre santé subjective mais a refusé de répondre sont exclus. Cet échantillon d'exclus comprend 706 enfants. Ces exclusions sont susceptibles de créer un biais dans nos estimations. Nous observons que cet échantillon d'exclus est similaire à l'échantillon inclus du point de vue du type de ménage, du genre et de l'âge des enfants, et de l'âge des parents. Mais ces enfants exclus vivent dans des familles aux revenus plus faibles et qui comptent plus de membres que les enfants inclus. Cependant, notons que la taille de cet échantillon d'exclus (706 enfants) est assez faible par rapport à l'échantillon d'inclus (6448), ce qui atténue la portée du biais.

Les détails sont disponibles auprès des auteurs. 